\title{
Das demokratische Paradox, Antagonismus und Agonie der globalen Ordnung: Chantal Mouffes Überlegungen zur internationalen Politik
}

\author{
Andreas Vasilache
}

Eingegangen: 15. Oktober 2019 / Überarbeitet: 20. Juli 2020 / Angenommen: 1. August 2020 / Online publiziert: 14 . September 2020

(C) Der/die Autor(en) 2020

Zusammenfassung Trotz der intensiven Diskussion poststrukturalistischer Ansätze in den Internationalen Beziehungen (IB) hat das sogenannte radikaldemokratische Denken bislang nur wenig Aufmerksamkeit erfahren. Radikaldemokratische Theoretiker*innen haben sich zugleich kaum mit Themen der internationalen Politik auseinandergesetzt. Eine Ausnahme bildet hier Chantal Mouffe, die sich in jüngerer Zeit unmittelbar mit Fragen der internationalen Politik befasst hat. Der vorliegende Artikel hat eine Bestandsaufnahme und Diskussion der Überlegungen Mouffes zur internationalen Politik zum Ziel. Mouffes diesbezügliche Reflexionen bauen im Wesentlichen auf ihrer Theorie des Politischen auf. Daher werden zunächst jene Aspekte ihrer Theorie dargelegt, auf die Mouffe in ihren internationalen Überlegungen rekurriert. Danach wird ihr auf dem Modell der agonistischen Politik fußender Vorschlag einer multipolaren Weltordnung besprochen. Hieran anschließend soll Mouffes Plädoyer für einen Pluralismus politischer Systeme und auch kulturspezifischer Menschenrechtsauffassungen thematisiert werden. In einem weiteren Schritt sind Mouffes Diagnose und Kritik einer Moralisierung der Politik zu erörtern, bevor ihre Skepsis gegenüber einer Ächtung des Krieges diskutiert wird. Der Artikel endet mit einigen einordnenden und ausblickhaften Bemerkungen zu Mouffes Ansatz in der internationalen Politik.

Schlüsselwörter Politische Theorie - Internationale Beziehungen (IB) · Internationale politische Theorie (IPT) · Poststrukturalismus $\cdot$ Konflikt

Dieser Aufsatz ist dem Andenken Rainer Schmalz-Bruns' gewidmet - als Mittler zwischen politischer Theorie und Internationalen Beziehungen, mehr aber noch als Freund.

A. Vasilache $(\square)$

Fakultät für Soziologie, Universität Bielefeld, Universitätsstraße 25, 33615 Bielefeld, Deutschland

E-Mail: Andreas.Vasilache@uni-bielefeld.de 


\title{
The Democratic Paradox, Antagonism, and Agonism of the Global Order: Chantal Mouffe's Considerations on International Politics
}

\begin{abstract}
Despite broad and ongoing interest in poststructuralist approaches in International Relations (IR), the so-called radical democratic thinking has so far received only little attention. At the same time, radical democratic theorists have barely addressed topics of international politics. Chantal Mouffe is an exception in this respect as she has been dealing directly with questions of international politics. This article discusses Mouffe's thoughts on international politics. Since her reflections are based on her theory of the political, we first outline those aspects of Mouffe's theory that she refers to in her considerations on international politics. Then we discuss her proposal for a multipolar world order that is based on the model of agonistic politics. This is followed by a consideration of Mouffe's suggestion of a pluralism of political systems and of culture-specific understandings of human rights. In a further step, Mouffe's diagnosis and criticism of the moralization of politics are discussed. Based on this, we then examine her skepticism of outlawing war as a means of politics. In the concluding section, some general and prospective remarks on Mouffe's approach to international politics are made.
\end{abstract}

Keywords Political theory · International relations (IR) · International political theory (IPT) · Poststructuralism · Conflict

\section{Einleitung: Mouffes (mittelbare) Rezeption in den Internationalen Beziehungen und ihre Hinwendung zur internationalen Politik}

Dass bislang noch kaum Auseinandersetzungen mit Chantal Mouffes Überlegungen zur internationalen Politik vorliegen, ist aus drei Gründen überraschend. Zum Einen hat in den letzten Jahren im Bereich der politischen Theorie, und dabei nicht zuletzt im deutschsprachigen Raum, eine breite und lebhafte Diskussion um Perspektiven der radikalen Demokratie stattgefunden. ${ }^{1}$ Mouffes Denken hat hier eine prominente Position eingenommen, zumal das gemeinsam mit Ernesto Laclau verfasste Buch Hegemony and socialist strategy (Laclau und Mouffe 2001) als ein einflussreicher, wenn nicht gar initialer Text für das zeitgenössische radikaldemokratische Denken gelten kann.

Zum Zweiten zeichnen sich die Internationalen Beziehungen (IB) - wie Herschinger feststellt - durch eine offene und ,rege Importpolitik“ (2017, S. 127) aus, ${ }^{2}$ wobei in den letzten Jahren an der Diskussion poststrukturalistischer Ansätze in den IB kein Mangel herrschte. ${ }^{3}$ Diese aber konzentrierten sich, neben Bezügen zu Der-

\footnotetext{
1 Vgl. z. B. Trend (1996); Little und Lloyd (2009); Heil et al. (2011); Priester (2014); Flügel-Martinsen und Marchart (2014); Tønder und Thomassen (2014); Rzepka und Straßenberger (2014); Marchart (2010, 2013); Flügel-Martinsen (2017, Kap. 3); Hetzel (2017); Nonhoff (2017); Comtesse et al. (2019).

${ }^{2} \mathrm{Ob}$ dies (sub)disziplinär eine Stärke ist, wird in jüngerer Zeit auch kontrovers diskutiert. Vgl. Rosenberg (2016).

3 Vgl. früh Edkins (1999). Für Überblicke über die vielfältige Rezeption poststrukturalistischen Denkens in den IB vgl. Edkins und Vaughan-Williams (2009) sowie aktuell Çalkıvik (2017).
} 
rida (vgl. z. B. Diez 1999; Plaxton 2000; Zehfuss 2002, S. 196-249), insbesondere auf die Fruchtbarmachung Foucaults (vgl. i.d.S. Herschinger 2017, S. 131) - und vor allem seiner späten, gouvernementalitätstheoretischen Überlegungen - für das Verstehen internationaler Politik. Während die Breite dieses Diskurses und die mittlerweile kaum mehr zu überblickende Literatur ${ }^{4}$ es bereits gerechtfertigt erscheinen lassen, von einer gouvernementalitätstheoretischen Strömung in den IB oder einer Foucauldian IR zu sprechen, sind radikaldemokratische Perspektiven nur vereinzelt auf Fragen der internationalen Politik bezogen worden. Wenn radikaldemokratische Ansätze in den IB aufgegriffen werden, dann wird meist auf Laclaus Denken rekurriert (vgl. i.d.S. Nonhoff 2017, S. 81). In der Befassung mit dem Werk Laclaus wird zwar, gleichsam ipso facto, immer dann auch auf Mouffe Bezug genommen, wenn Hegemony and socialist strategy (Laclau und Mouffe 2001) thematisch wird. ${ }^{5}$ Doch bleibt dies eine indirekte, vornehmlich auf einen einzelnen (wenngleich prominenten) Text begrenzte Berücksichtigung des Mouffe'schen Denkens. Zu einer unvermittelten Beschäftigung mit der Theorie Mouffes, d.h. jenseits ihrer Beteiligung an der Essex School, hat dies in den IB jedenfalls bislang nicht geführt. ${ }^{6}$

Die geringe und nur gelegentliche unmittelbare Befassung mit Mouffe in den IB überrascht zum Dritten schließlich auch deshalb, weil sich Mouffe - im Unterschied zu anderen radikaldemokratischen und poststrukturalistischen Denkern wie Nancy, Rancière, Lefort oder auch Laclau und Foucault ${ }^{7}$ - seit einigen Jahren dezidiert mit Fragen der europäischen und internationalen Politik befasst. Insbesondere hat sie ihren Begriff des Politischen und der Hegemonie sowie ihren theoretischen Ansatz der Agonistik auf die internationale Politik bezogen und hieraus Vorschläge für eine agonistische globale Ordnung (vgl. Mouffe 2007, Kap. V, 2010, S. 115-118, 2014, Kap. 2, 3) entwickelt. Aus dieser theoretischen Perspektive hat sie sich mit dem Verhältnis zwischen Uni- und Multipolarität, mit der Kritik des liberalen Demokratieund Menschenrechtsuniversalismus, mit Fragen von Hegemonie, Systemkonkurrenz und Krieg sowie mit dem Verhältnis zwischen politischen und moralischen Rechtfertigungs- und Handlungslogiken in der internationalen Politik auseinandergesetzt. ${ }^{8}$

Nun ist die bisher geringe Beachtung der Überlegungen Mouffes zur internationalen Politik nicht nur verwunderlich, sondern kann auch als Rezeptionslücke

\footnotetext{
${ }^{4}$ Vgl. für einen breiteren Literaturüberblick Vasilache (2019a).

5 In den Rekursen auf Laclau sowie auf Laclau und Mouffe in den IB spielen Diskurse und ihre Verschränkung mit kollektiven Identitäten (vgl. Laclau und Mouffe 2001, Kap. 3; Laclau 1995) eine hervorgehobene Rolle. So besteht ein wichtiger Beitrag bisheriger Rezeptionen darin, theoretische und konzeptionelle Bezüge für die Diskursforschung in den IB zu liefern. Vgl. bereits Campbell (1998, S. 6); Diez (1999) sowie für die in jüngerer Zeit intensivere Rezeption Diez (2014); Herschinger (2017); Herschinger und Renner (2014); Wenman (2009); Nabers (2015); Wojczewski (2018). Vgl. auch das Symposium zu Laclau in der Zs. New Political Science 41(2) sowie darin Stengel und Nabers (2019).

${ }^{6}$ Vgl. für vereinzelte Rekurse auf Teilaspekte der politischen Theorie Mouffes Kapoor (2002); Tsakatika (2007); Conway und Singh (2011).

7 Dagegen hat sich auch Derrida (1994a, 1994b, 2003) mit Fragen der internationalen Politik befasst.

8 Die bislang geringe Rezeption der internationalen Überlegungen Mouffes mag auch dieser Divergenz geschuldet sein zwischen Mouffes Interesse an den internationalen Konsequenzen des antagonistischen Charakters des Politischen einerseits und den v. a. diskurstheoretisch und konzeptionell orientierten Bezugnahmen auf das radikaldemokratische Denken in den IB andererseits, die daher schwerpunktmäßig auf Laclau und dabei auf Mouffe lediglich als Co-Autorin rekurrieren.
} 
gelten. Es sind in thematischer und systematischer Hinsicht fünf miteinander verflochtene Gründe zu nennen, die eine Befassung mit Mouffes Nachdenken über die internationale Politik lohnenswert machen und den Mehrwert einer Auseinandersetzung mit ihrem Denken begründen. Erstens ist angesichts des Einflusses Mouffes in aktuellen Diskursen der Radikaldemokratie, in Anbetracht der Fruchtbarkeit poststrukturalistischer Ansätze in den IB sowie schließlich aufgrund der unmittelbaren Zuwendung Mouffes zur internationalen Politik eine Rezeption ihrer internationalen Überlegungen schon mit dem Ziel einer Theoriediskussion geboten. Darüber hinaus sprechen vor allem folgende Aspekte - die gleichsam als Thesen den vorliegenden Text anleiten - für eine Berücksichtigung ihres internationalen Nachdenkens. Denn zweitens setzt sich Mouffe mit thematischen Eckpfeilern und wesentlichen Fragen der globalen Ordnung und daher gleichsam mit den großen und klassischen Themen der internationalen Politik und der IB auseinander. Während jeder von Mouffe andressierte Themenkomplex schon für sich genommen gewichtig ist, werden diese Themenbereiche aber drittens nicht nur gesondert oder parallelisiert besprochen, sondern eng miteinander verwoben sowie im Lichte ihres agonistischen Modells der Politik konflikt- und machtanalytisch fokussiert. In diesem Sinne zeichnet sich Mouffes Zugang zur internationalen Politik viertens auch durch eine untrennbare Verschränkung von empirischer und theoretischer Perspektivierung aus. Wenngleich es im Folgenden nicht um eine disziplinäre Verortung Mouffes zwischen politischer Theorie und IB oder um die Frage der Konstituierung einer internationalen politischen Theorie gehen wird, besteht hier doch ebenfalls ein Potenzial ihrer Ausführungen zur internationalen Politik. Nicht zuletzt aus der Verschränkung eines empirischen und theoretischen Zugriffs ergibt sich schließlich fünftens Mouffes Ziel, konkrete und praktische Empfehlungen für die internationale Politik und die globale Ordnung auszusprechen. Wie sich zeigen wird, verdichtet sich ihre Perspektive dabei zu einem normativen Realismus.

Der vorliegende Artikel soll einen Beitrag zu einer ersten Gesamtschau und kritischen Bestandsaufnahme der Ausführungen Mouffes zur internationalen Politik leisten. Aufgrund der in den IB wenig ausgeprägten Bezugnahme auf Mouffe kann der Schwerpunkt dabei nicht auf ihrem dortigen Einfluss liegen. Vielmehr sollen - selbst wenn die Unterscheidung zwischen IB, internationalen Beziehungen und internationaler Politik nie ganz trennscharf ist (vgl. noch immer Czempiel 2004) - Mouffes Vorschläge zum Verständnis und zur Kritik der internationalen Politik dargestellt und diskutiert werden. Deshalb werde ich mich im Wesentlichen auf jene jüngeren Texte ihres Werkes konzentrieren, in denen sie sich explizit mit internationalen Fragen und Herausforderungen befasst. Da Mouffes Denken des Internationalen auf ihrer politischen Theorie und ihrer Theorie des Politischen aufbaut, wird gleichwohl auch auf weitere Texte zu rekurrieren sein.

Der Aufbau des vorliegenden Beitrags rekonstruiert und folgt den thematischen Schwerpunkten, die Mouffe in ihren Überlegungen zur internationalen Politik setzt. Weil ihr Nachdenken über die internationale Politik vornehmlich auf ihrem konfliktiven Begriff des Politischen aufbaut, soll zunächst ein Abriss ihrer Theorie des Politischen sowie der agonistischen Demokratie gegeben werden (Abschnitt 2), um dann ihren Vorschlag einer multipolaren Weltordnung konkurrierender Hegemonialmächte zu diskutieren (Abschnitt 3). Anschließend soll Mouffes Plädoyer für einen 
globalen Pluralismus politischer Systeme und kulturspezifischer Menschenrechtsauffassungen thematisiert werden (Abschnitt 4). In einem weiteren Schritt ist ihre Kritik einer Moralisierung der Politik zu besprechen (Abschnitt 5). Hieran anknüpfend wird ihre Skepsis gegenüber einer Ächtung des Krieges zu diskutieren sein (Abschnitt 6), bevor abschließend einige allgemeinere Implikationen ihres internationalen Nachdenkens ausgewiesen werden sollen (Abschnitt 7).

\section{Eine politische Theorie des Politischen: Demokratisches Paradox, Antagonismus und Agonistik}

Nähert man sich Mouffes Überlegungen zur internationalen Politik, so fällt zunächst ins Auge, dass ihre thematische wie theoretische Schwerpunktsetzung nicht an die in den IB bereits vorliegenden Diskussionen des mit Laclau verfassten Buches anknüpft. Ihre mit Laclau vorgelegte Theorie bildet im Bereich der Diskursforschung in den IB - und dabei nicht zuletzt mit Blick auf diskursive Identitätskonstruktionen in der internationalen Politik - einen wichtigen und produktiven Bezugspunkt (vgl. Diez 2014; Herschinger und Renner 2014). Im Unterschied hierzu stehen bei Mouffe nun aber der Begriff des Politischen sowie seine Konsequenzen für konkrete Herausforderungen der internationalen Politik im Zentrum des Interesses. Ihre Diskussionen zur internationalen Politik basieren zwar auf dem mit Laclau erarbeiteten Antagonismusverständnis. Ansonsten aber bezieht sich Mouffe kaum direkt auf die - nicht zuletzt im Kontext der Essex School formulierten - Konzepte zur hegemonialen Konstruktion von Subjektivitäten und Identitäten. Vielmehr nehmen ihre Ausführungen zum Internationalen ihren theoretischen Ausgang im Begriff des Politischen sowie in ihren Überlegungen zum demokratischen Paradox, zur ,politische[n] Differenz" (Marchart 2010, S. 15, vgl. insgesamt Marchart 2010) sowie zur Agonistik und ihrem agonistischen Modell. So weist Mouffe (2010, S. 20) aus, dass die liberale Demokratie

aus der Artikulation zweier unterschiedlicher Traditionen resultiert. Auf der einen Seite haben wir die liberale Tradition, die von [...] individueller Freiheit gekennzeichnet ist, auf der anderen die demokratische Tradition, deren Hauptideen jene der Gleichheit [...] und der Volkssouveränität sind.

Es gebe allerdings ,kein notwendiges Verhältnis zwischen diesen beiden unterschiedlichen Traditionen“ (Mouffe 2010, S. 20). Vielmehr bestehe die „paradoxe Natur" (Mouffe 2010, S. 22) der liberalen Demokratie darin, dass diese beiden Leitideen ,,in letzter Instanz inkompatibel sind“ (Mouffe 2010, S. 22) und die „konstitutive Spannung [...] nie aufgelöst, sondern immer nur auf unterschiedliche Weise verhandelt werden kann" (Mouffe 2010, S. 22). Da aus Mouffes Sicht dieses Spannungsverhältnis nicht nur unüberwindbar, sondern für die liberale Demokratie konstitutiv ist, wird schon durch das Ziel seiner Überwindung die Möglichkeitsbedingung liberaler Demokratie untergraben. Der Vorstellung eines rationalen, normativ fundierenden Konsenses, der den Konflikt zwischen freiheitlich-liberaler und volksdemokratisch-egalitärer Tradition überwinden könnte, erteilt sie daher eine klare Absage. Eine solche harmonisierende Vorstellung, wie sie auch in der Haber- 
mas'schen Idee deliberativer Demokratie zum Tragen komme (vgl. Mouffe 2000; vgl. auch Kapoor 2002; Flügel-Martinsen und Marchart 2014, S. 199), ist aufgrund der konstitutiven Dichotomie von Freiheits- und Gleichheitslogik Mouffe zufolge nicht nur kein erstrebenswertes Ideal, sondern vielmehr eine Gefahr für den Bestand liberaler Demokratie selbst (vgl. 2010, S. 24, 27, 31, 46, 104, 106).

Dem sowohl unrealisierbaren als auch gefährlichen Irrweg einer Auflösung dieser konfliktiven Spannung setzt Mouffe die Notwendigkeit der Anerkennung der paradoxen Natur liberaler Demokratie entgegen (vgl. 2010, S. 26-27, 31-32). Erst durch die Anerkennung der Unüberwindbarkeit und der konstitutiven Funktion des Widerstreites zwischen Freiheits- und Gleichheitslogik werde es möglich, ,die wirklichen Stärken liberaler Demokratie“ (Mouffe 2010, S. 26) zu erkennen und in praxi zu realisieren. In anlehnender Abgrenzung zu Schmitt (vgl. 2002, S. 26-36) hebt sie hervor, dass diese Spannung nicht notwendig die Form einer antagonistischen Konfrontation zwischen Freund und Feind annehmen müsse, ,die keinen gemeinsamen symbolischen Raum teilen“ (Mouffe 2010, S. 29). Vielmehr könne sie in agonistischer Weise ausgetragen werden, die „kein Verhältnis zwischen Feinden beinhaltet, sondern eines zwischen ,Gegnern', die auf paradoxe Weise als ,freundschaftliche Feinde" definiert werden“ (Mouffe 2010, S. 29-30). Der Gegner bleibt Mouffe (2010, S. 103) zufolge

ein Feind, doch ein legitimer Feind, mit dem wir [...] eine gemeinsame Bindung an ethisch-politische Prinzipien liberaler Demokratie besitzen: Freiheit und Gleichheit. Aber wir stimmen nicht überein, was die Bedeutung und Implementierung dieser Prinzipien betrifft [...].

Dabei gebe es in diesem „agonistischen Pluralismus“ (Mouffe 2010, S. 104) „keine rationale Lösung des Konflikts“ (Mouffe 2010, S. 104). Vielmehr sei jede Übereinkunft ein spezifischer, angreifbarer und zeitlich begrenzter ,konfliktorischer Konsens“ (Mouffe 2010, S. 105; vgl. Rzepka und Straßenberger 2014), der sich aus dem unentwegten Bestreben der ,,verschiedenen Interpretationen der ethisch-politischen Prinzipen“ (Mouffe 2010, S. 105) ergebe, „eine unterschiedliche Form von Hegemonie zu implementieren“ (Mouffe 2010, S. 105). In diesem Zusammenhang kommt auch die Bedeutung politischer Institutionen in Mouffes Ansatz zum Tragen (vgl. hierzu Wallaschek 2017; Westphal 2019). Denn die agonistische Umwandlung antagonistischer Konfrontationen hat ihren Raum in - immer kontingenten politischen Institutionen, in denen das eigene wie das gegnerische Streben nach diskursiver und politischer Hegemonie realisiert werden müsse (vgl. Mouffe 2007, S. 157, 2010, S. 37, 2014, S. 12, 14, 18, 23, 195).

In politischen Ansätzen des sogenannten dritten Weges oder einer Politik der Mitte, die die Möglichkeit und Notwendigkeit der Überwindung des Konflikts zwischen Freiheit und Gleichheit hervorheben, erkennt Mouffe dagegen eine ,gefährlich[e] Versöhnungsutopie“ (2010, S. 43; vgl. auch Tambakaki 2009) - und letztlich das neoliberal geprägte Hegemoniestreben des liberalen Prinzips, das in seinem aktuellen Erfolg die Legitimität der Artikulation von Dissens, insbesondere seitens des egalitären, volksdemokratischen Prinzips, in Zweifel ziehe. Damit aber werde die Grundlage liberaler Demokratie selbst untergraben sowie die Möglichkeit agonistischer Konflikteinhegung innerhalb politischer Institutionen unterminiert (vgl. 
Mouffe 2007, S. 10-11, 2010, S. 31-32, 47, 112, 128, 131), wodurch die Gefahr der Schmitt'schen, antagonistischen Konfliktform einer irreduziblen Dichotomie von Freund und Feind (Schmitt 2002, S. 26-36, 45-58, 78) ansteige.

Erst die Überwindung der „Blindheit für den Antagonismus“ (Mouffe 2007, S. 8) und die Einsicht in den für das Politische konstitutiven Charakter von Konflikten, „für die es niemals eine rationale Lösung geben kann“ (Mouffe 2007, S. 17), eröffnen Mouffe zufolge die Möglichkeit einer widerständigen, progressiven Politik (vgl. Flügel-Martinsen und Marchart 2014, S. 198-200). Daher spricht sie sich für die Anerkennung eines machtgesättigten agonalen Dissenses - sowie schließlich für „die Bildung einer neuen Hegemonie“ (Mouffe 2007, S. 71, 2018) - aus, die das liberale Prinzip von einem egalitär-volksdemokratischen Standpunkt aus angreife (vgl. Mouffe 2007, S. 37-38, 71, 2010, S. 114-121, 124, 2018). So ergibt sich nach Mouffe aus der konflikthaften Konstitution des Politischen die Notwendigkeit der Verfolgung neuer, alterierender und gegenläufiger hegemonialer Diskurse und Bestrebungen, die nicht als konsensuelle bzw. nichthegemoniale verbrämt, sondern als agonistische, gegenhegemoniale Bewegungen verfasst sein müssen.

\section{Die internationale Ordnung politisch denken: Agonistische Multipolarität und Hegemonialkonkurrenz}

Die Überlegungen Mouffes zur internationalen Politik setzen mit einer einschränkenden Bemerkung ein. Mouffe hebt hervor, keine reine Anwendung ihrer Theorie im Bereich der IB ,im Wortsinne“ (2014, S. 50) vornehmen zu wollen, da ,die Lage auf der nationalen und der internationalen Ebene grundverschieden“ (2014, S. 50) sei. Zugleich aber diagnostiziert Mouffe, dass sowohl auf der innenpolitischen als auch auf der internationalen Ebene das zentrale Problem in der Verkennung der grundsätzlich konflikthaften Struktur des Politischen zugunsten eines naiven wie gefährlichen Konsensideals bestehe (vgl. 2007, S. 107-108, 2014, S. 45-51). Aufgrund der aus Mouffes Sicht gleichen Problemlage treten ihre kurz formulierten Bedenken gegen eine Applikation ihrer Theorie im Bereich der internationalen Politik schließlich vollständig in den Hintergrund. In der Folge steht die - nur leicht adaptive - Übertragung ihres agonistischen Politikmodells auf die internationale Ebene im Mittelpunkt ihrer Ausführungen zur internationalen Politik.

Basierend auf ihrem antagonistischen Begriff des Politischen und ihrem Konzept einer agonistischen Politik stellt Mouffe nun die Frage nach den Merkmalen und der Verfasstheit einer globalen Ordnung, die den grundsätzlich konflikthaften, machtund hegemonieorientierten Charakter des Politischen nicht zu überwinden sucht, sondern einen Rahmen für eine friedliche, nichteskalative Austragung politischer Antagonismen breitstellen kann (vgl. 2007, Kap. V, 2014, Kap. 2). Die mit diesem Modell korrespondierende Struktur der internationalen Politik erkennt Mouffe in einer multipolaren Ordnung, in der die aktuelle Hegemonie des westlich-liberalen Systems durch weitere regionale Pole herausgefordert werden würde. In empirischer Hinsicht bildet dabei insbesondere der liberale Interventionismus der USA seit den 1990er-Jahren für Mouffe die Kontrastfolie zu ihrem Vorschlag einer Ordnung multipolarer Hegemonialkonkurrenz (vgl. 2007, S. 151-155, 2014, S. 46-51). Die „ex- 
plosionsartige Entstehung neuer Antagonismen, die in der Tat zum vorausgesagten ,Kampf der Kulturen“ führen könnten“ (Mouffe 2007, S. 151), ist aus ihrer Sicht das Resultat einer unangefochtenen, unipolaren Hegemonie der USA und ihrer Verbündeten. Das „Fehlen politischer Kanäle, über die sich die Hegemonie des neoliberalen Globalisierungsmodells infrage stellen ließe“ (Mouffe 2014, S. 45), führe zur Entladung bestehender Antagonismen in der gewaltförmigen Logik einer Freund-FeindDichotomie und stehe einer agonistischen Einhegung von Antagonismen im Wege (vgl. Mouffe 2007, S. 107-108, 2014, S. 50). Eine solche agonistische Einhegung ist nach Mouffe dagegen durch eine multipolare Balance mehrerer hegemonialer Pole erreichbar, „die nach unterschiedlichen wirtschaftlichen und politischen Modellen organisiert sind, ohne dass es eine zentrale Autorität gäbe“ (2014, S. 49, vgl. auch S. 72-73). Durch die Überwindung unipolarer Hegemonie zugunsten agonistischer Multipolarität sei ,die Möglichkeit des legitimen Dissenses“ (Mouffe 2014, S. 46) wieder gegeben und es hielten sich, so könnte man sagen, die unterschiedlichen Pole gegenseitig in Schach, wodurch, die Wahrscheinlichkeit sänke, dass diese Konflikte eine antagonistische Form annehmen“ (Mouffe 2014, S. 50).

Mouffe weist ausdrücklich darauf hin, dass eine solche multipolar geordnete, agonale Welt nicht nur aus Demokratien oder demokratischen Polen zusammengesetzt wäre (vgl. 2014, S. 57). Zur Abwendung des offenen Ausbruchs vorhandener Antagonismen zieht Mouffe dies aber zum einen der diagnostizierten unipolaren Situation vor und weist zum anderen die Vorstellung eines universellen Geltungsanspruchs der liberalen Demokratie und überhaupt eines „Konsens[es] auf der Basis eines einzigen Modells“ (2014, S. 46) als expansionistisch und daher illegitim und gefährlich zurück (vgl. 2007, S. 113-114, 2014, S. 49-50, 56, 62-63; vgl. Flügel-Martinsen und Marchart 2014, S. 200). Sehr deutlich wird hier, dass Mouffe ein zwar nicht auf bestimmte realistische Schulen rekurrierendes, aber doch klar realistisch geprägtes Verständnis internationaler Politik vertritt, das in seiner Interessens- und Konfliktorientierung sowie seiner ausgeprägten Skepsis gegen moralbasierte und wertegeleitete Ansätze in der internationalen und Außenpolitik (vgl. 2007, S. 11-12, 100; vgl. i.d.S. auch Schmitt 2002, S. 37) mit dem Grundbestand realistischen Denkens korrespondiert. ${ }^{9}$ Der Anspruch Mouffes, der konstatierten liberal-kosmopolitischen Hegemonie eine - auch im Sinne politischer Praxis - realistische Perspektivierung des Internationalen entgegenzusetzen, wird auch offenkundig, wenn sie kritisiert, dass ,die optimistische Anthropologie auch heute noch die vorherrschende“ (2007, S. 9) sei - was angesichts der langen Tradition und des handlungsleitenden Einflusses des (neo-)realistischen Denkens in den IB und der internationalen Politik allerdings kaum zu belegen sein dürfte. Eine realistische Vorstellung internationaler Politik zeigt sich ebenfalls in ihrer Warnung vor einer Idealisierung internationaler Organisationen, deren Kompetenzzuwachs zulasten nationaler Demokratien sie kritisiert (vgl. Mouffe 2007, S. 136-138). Mouffes realistisches Verständnis internationaler Politik offenbart sich schließlich auch in der Feststellung, dass ,das Einzige, worauf man im Bereich der internationalen Beziehungen zählen kann, kluge Verträge sind“" (2014, S. 51). Dieser notwendigen Einsicht stünden dagegen zum einen

\footnotetext{
9 Vgl. z. B. Niebuhr (1934, S. 231-236, 256); Carr (1964, Kap. 7-9); Morgenthau (1993, S. 4-16); Waltz
} (2010, S. 194-210); Mearsheimer (2014, S. 12-27). 
die „Illusion von einer globalen Ethik, einer globalen Zivilgesellschaft und andere kosmopolitische Träume“ (Mouffe 2014, S. 51) im Wege. Zum anderen sieht sie ebenfalls Vorstellungen einer kollektiven, kontraktualistischen Überwindung der globalen Anarchie als irreführend an, sodass ,[a]lle Versuche, durch ein globales Abkommen den ,Naturzustand" zwischen Staaten ein für alle Mal zu überwinden“ (Mouffe 2014, S. 51) als undurchführbar und gefährlich abzulehnen seien (vgl. Mouffe 2014, S. 49-50, 56-57).

Nun lässt Mouffe allerdings offen, weshalb die Zurückweisung der - ihr zufolge letztlich immer imperialistischen - Durchsetzung vermeintlich universeller, liberaler Ordnungsmuster ${ }^{10}$ sowie die Ablehnung der Utopie eines Weltstaates einerseits und der Versuch der Konstituierung und schrittweisen Etablierung verbindlicher grenzüberschreitender Normen andererseits sich zwingend ausschließen müssten. Entgegen einer solchen dichotomen Perspektivierung scheint vielmehr gerade in Ermangelung einer weltstaatlichen Option die Arbeit an grenzüberschreitenden und internationalen Normen und Standards - ganz im Sinne eines ,konfliktualen Konsens[es]“ (Mouffe 2007, S. 158), der der „Irreduzibilität des Konflikts der Werte“ (Mouffe 2007, S. 159, vgl. 2014, S. 72-73) Rechnung trägt - der Mühe wert zu sein. Dass aus Mouffes Sicht die Voraussetzung für einen solchen konflikthaften Konsens aufgrund der Inexistenz ,einer politischen Gemeinschaft [...] auf globaler Ebene" (2014, S. 51) nicht gegeben sei, leuchtet zwar mit Blick auf die von ihr kritisierte Vision eines Kosmopolitismus ohne Staaten, d.h. auf der interpersonalen und inter(zivil)gesellschaftlichen Beziehungsebene, ein. Doch schließt diese Kritik die Möglichkeit und das Ziel eines solchen konflikthaften Konsenses auf der von Mouffe für die internationale Politik ja favorisierten Ebene der zwischenstaatlichen Beziehungsmuster nun gerade nicht aus. Versuche der internationalen Normgenerierung - deren Ergebnisse immer umstritten und provisorisch sein sowie in der internationalen Sphäre als Soft Law eine geringere Verbindlichkeit haben werden als im innerstaatlichen Bereich - müssen jedenfalls auch aus Mouffes Ansatz heraus keineswegs zwingend als „kosmopolitische Träume“ (2014, S. 51) disqualifiziert werden.

Mouffes ausgeprägte Skepsis gegenüber kosmopolitischen und konsensorientierten Rationalitäten in der internationalen Politik folgt auch daraus, dass sie sie nicht nur als theoretischen Irrweg erkennt, sondern als in der politischen Praxis dominant ansieht. So geht Mouffe in ihrer Kritik an liberalen Ansätzen und in ihrer Forderung eines agonal-konfliktiven Gegenmodells von der empirischen Voraussetzung aus, dass die internationale Politik und ihre Strukturen vornehmlich durch ein westlichliberales Konsensideal globaler Demokratisierung und supranationaler Governance im Rahmen von internationalen Organisationen sowie durch die fehlende Anerkenntnis und die Unsichtbarkeit konfliktträchtiger Gegensätze charakterisiert seien. Diese Voraussetzung muss aber als fraglich gelten - schon allein deswegen, weil Struktu-

\footnotetext{
$10 \mathrm{Vgl}$. zum Einfluss und auch zur Instrumentalisierung liberaldemokratischer Argumente im liberalen Interventionismus z. B. Chandler (2006); Ish-Shalom (2008); Reus-Smit (2005); Sørensen (2006). Für das Verhältnis zwischen liberalen und illiberalen Räumen in liberalen Ordnungsvorstellungen vgl. Jahn (2018) und für den Illiberalismus des liberalen Interventionismus Desch (2008). Vgl. für einen Überblick über die gouvernementalitätstheoretische Diskussion in den IB zum (il)liberalen Regieren Vasilache (2019a, S. 688-689).
} 
ren und Dynamiken der internationalen Politik und innerhalb internationaler Organisationen keineswegs von einem Übermaß an (westlich-hegemonial produzierter) konsensueller Harmonie oder überhaupt von einem unipolar als universell durchgesetzten liberaldemokratischen Mainstream gekennzeichnet sind. Weder herrscht in der internationalen Politik in praxi ein Mangel an offensichtlichen - antagonistisch wie agonistisch ausgetragenen - Konflikten, noch lässt sich im Bereich des theoretischen Nachdenkens über internationale Politik eine diskursive Hegemonie kosmopolitischer oder konsensorientierter Globalismusansätze feststellen. Und gerade auch die in den IB intensiv geführte Diskussion um liberale Weltordnungsmodelle und ihren (vermeintlichen) Niedergang lässt sich aufgrund der Differenziertheit ihrer Perspektivierungen, Diagnosen und Ausblicke keineswegs als uniforme Affirmation eines liberalen kosmopolitischen Globalismus zusammenfassen. ${ }^{11}$ Durch Mouffes ausschnittsweise Betrachtung einzelner weniger kosmopolitischer Ansätze kann der theoretisch-konzeptionelle oder praxisleitende hegemoniale Erfolg dieser Ansätze auf globaler Ebene jedenfalls nicht nachgewiesen werden.

In Mouffes Ausweis und Kritik einer westlich-liberalen Hegemonie ist zudem ein systematisches Spannungsverhältnis festzustellen. Dieses Spannungsverhältnis besteht darin, dass sie die Demokratisierungsmission des westlichen Liberalismus einerseits als Ausdruck eines partikularen, westlichen Hegemoniestrebens - mit allen militärischen und damit antagonistisch-gewaltförmigen Eskalationen im Rahmen des liberalen Interventionismus - kritisiert sowie andererseits und zugleich als Konsequenz und Ausdruck eines bereits erfolgreich durchgesetzten unipolaren kosmopolitischen Konsensideals ansieht. Angesichts der auch gewaltförmig ausbrechenden Antagonismen kann beides zugleich allerdings nicht der Fall sein. Die Feststellung des Partikularismus des kosmopolitischen Modells und seiner Instrumentalisierung im Rahmen westlichen Hegemonialstrebens steht in einem Widerstreit zur Behauptung einer bereits weitgehend unangefochten bestehenden globalen Hegemonie des westlichen liberal-kosmopolitischen Modells.

In diesem Zusammenhang muss Mouffes Diagnose einer unipolaren globalen Ordnung unter US-amerikanischer Hegemonie auch strukturell und empirisch hinterfragt werden. Zwei Einwände sind hier zu formulieren. So beschreibt Mouffe die von ihr festgestellte unipolare Vormacht der USA erstens ausschließlich als eine Situation globaler, gleichsam überwältigender Dominanz (vgl. 2007, S. 106, 151, 154, 2014, S. 49) - in der vom hegemonialen Zentrum letztlich nur Push-Faktoren ausgehen. Auf der Grundlage von alleine ,negativen Folgen [...] einer unipolaren Welt“" (Mouffe 2014, S. 49) lässt sich das erfolgreiche Bestehen einer einzigen, globalen Hegemonialmacht allerdings nur schwer erklären. Vor allem Münkler hat in seinem systematischen und vergleichenden Zugriff auf imperiale Ordnungsmuster gezeigt, dass ein unipolares Zentrum allein mit negativen Anreizen und Wirkungen, d.h. ohne die Produktion auch von Pull-Faktoren in Form von „GovernanceLeistung“ (2019, S. 71) - wie z. B. Ordnungsversprechen, Schutzzusagen, die Be-

11 Es sei z. B. auf den Band von Dunne und Flockhart (2013) hingewiesen, der aufzeigt, dass liberale Weltordnungsmodelle sich deutlich voneinander unterscheiden, sowie auf Zürns Differenzierung von Modellen und Etappen globaler liberaler Ordnungsbildung (2018, S. 223-230). Vgl. auch Ikenberry (2011); Duncombe und Dunne (2018, S. 41-42); Flockhart (2016); Jahn (2018). 
reitstellung öffentlicher Güter, Marktzugängen -, nicht auskommt. Auch imperiale Mächte bedürfen der Outputlegitimation, sodass ausschließlich negative und repressive Potenziale und Wirkungen für ihren Bestand und Erfolg nicht ausreichend sind (vgl. insgesamt Münkler 2019). Wenn Mouffe von ,der unzweifelhaften Überlegenheit der Vereinigten Staaten“ (2007, S. 154) als „der Hegemonie einer Hypermacht“ (2014, S. 49) spricht, dann ist zweitens - und grundsätzlicher - einzuwenden, dass die USA weder sicherheits- noch wirtschaftspolitisch als alleiniger globaler Pol oder einziges Zentrum der globalen Ordnung gelten können. Die Forderung nach einer Überwindung des aktuellen Zustands der US-dominierten liberalen Unipolarität ist schon hinsichtlich ihrer empirischen Diagnose zweifelhaft. Dieser Diagnose ist beispielhaft Acharyas (vgl. 2014, S. 12-58) Ausweis einer bestehenden weltregionalen Multipolarität sowie sein Argument entgegenzuhalten, dass liberale Ordnungsvorstellungen immer schon lokal eingebettet waren und auch kontestiert worden sind. ${ }^{12}$ Nicht zuletzt aber verkennt Mouffe sowohl das ökonomische Gewicht der EU oder Chinas als auch das sicherheitspolitische - und dabei auch nukleare - Gewicht Chinas oder Russlands, das den außenpolitischen Handlungsoptionen der USA und ihrer Interessensdurchsetzung regelmäßig Grenzen setzt, sei es im südchinesischen Meer, auf der koreanischen Halbinsel, im Kaukasus oder in Syrien. ${ }^{13}$ Dass US-Regierungen - nicht erst seit dem Amtsantritt des aktuellen Präsidenten, sondern seit den 1990er-Jahren - verstärkt zu unilateralem Handeln neigen, darf nicht mit Unipolarität verwechselt werden. Unilateralismus und Unipolarität sind unterschiedliche Phänomene, was freilich in Fällen des Misserfolgs unilateralen Handelns besonders offensichtlich wird. ${ }^{14}$

Wenn Mouffe eine auf multipolare Hegemonieansprüche basierende internationale Ordnung geltend macht, da diese dem agonalen Modell besser entspreche als ein liberales, auf Kompromiss und Konsens ausgerichtetes Ideal, dann greift dies allerdings auch in theoretischer wie konzeptioneller Hinsicht zu kurz. Nicht nur findet eine verkürzte Identifizierung von liberalen, kosmopolitischen Theoriebeiträgen mit einer vermeintlichen globalen Hegemonie des US-geführten (Neo-)Liberalismus statt. Vielmehr besteht ein wesentliches Problem auch darin, dass Mouffe den am Modell der diskursiven Auseinandersetzung erarbeiteten Hegemoniebegriff recht umstandslos mit dem Phänomen der Hegemonie in der internationalen Politik gleichsetzt. Dabei aber zeigt sich, dass ihre auf Gramsci rekurrierende und in abgrenzender Anlehnung an Schmitt (vgl. 2002, S. 26-36) fortentwickelte theoretische

\footnotetext{
12 Auch Acharya (vgl. 2014, S. 34) bezieht sich auf Gramsci, liest ihn aber hegemoniekritisch, während Mouffe das Hegemonialstreben als für das Politische notwendig erachtet und mit dem Ziel einer Gegenhegemonie positiv wendet.

${ }^{13}$ Vgl. für die Diskussion zur Bedeutung von Rising Powers für liberale Weltordnungsvorstellungen Flockhart (2016) und Zürn (2018, S. 171-192) sowie mit einem diesbezüglichen Blick auf Regionen Hurrell (2009, S. 214, 248-252).

${ }^{14}$ Bemerkenswerterweise zeigt sich die aktuelle multipolare globale Ordnung seit dem Amtsantritt des Unilateralisten Trump besonders eindrücklich. Denn schon die Geschwindigkeit, mit der andere Staaten jene Einflussbereiche füllen, die der Rückzug der USA z.B. aus Syrien oder auch aus internationalen Organisationen und Regimen hinterlässt, demonstriert den Bestand und die Handlungsfähigkeit anderer Pole. Und auch die (nukleare) Sicherheitsgarantie der USA für West- und Mittelosteuropa zeugt freilich von der diesbezüglichen Abhängigkeit Europas von den USA angesichts einer multipolaren Ordnung, nicht aber von globaler Unipolarität.
} 
Einsicht, wonach durch die Anerkenntnis des Kampfes um diskursive Hegemonie potenziell gewaltförmige Antagonismen als agonale Konflikte einzuhegen sind, nicht ohne Weiteres auf die internationale Ebene übertragen werden kann. Denn während Mouffe auf ihr in früheren Schriften entwickeltes Konzept der diskursiven Hegemonie rekurriert, bleiben die spezifischen Strukturen internationalen, staatlichen Hegemonialstrebens unterbelichtet. So entgehen Mouffes vornehmlich diskursivem Hegemoniebegriff ${ }^{15}$ die besonderen und immer auch materialen Voraussetzungen und Implikationen internationaler Hegemonieansprüche. Außenpolitische und internationale Hegemonieansprüche und -praktiken kommen bisweilen mit einem nur geringen $\mathrm{Ma}$ an diskursivem Hegemoniepotenzial aus und rekurrieren auf andere Ressourcen. Zwar ist Mouffe Vertreterin eines nicht alleine auf Sprache bezogenen, sondern breiten Diskursbegriffs, der im Anschluss an Gramsci und Foucault auch nichtsprachliche Praktiken und Techniken inkludiert (vgl. Mouffe und Laclau 2001, S. 67, 105-111, 134-135, 145). Gleichwohl kommt in der Anwendung ihres Konzepts der Hegemonie auf die internationale Politik und in dem hieraus resultierenden Plädoyer für eine multipolare Ordnung konkurrierender Hegemonien vornehmlich eine diskursiv-ideelle sowie diskursiv-sprachliche, auf konkurrierende Wert- und Weltvorstellungen verweisende Dimension von Hegemonie zur Geltung. Hingegen bleiben die strukturellen, materialen und insbesondere die militärischen Aspekte internationaler Hegemonie und Hegemonialstrebens weitgehend unbeachtet. Dabei ist das Problem der strukturellen Instabilität und der militärischen Eskalationstendenz internationaler Hegemonialkonkurrenz vor allem dann von besonderer Relevanz und hätte gleichsam erst recht hervorgehobener Aufmerksamkeit bedurft -, wenn man im Anschluss an Mouffe die internationale Politik in realistischer Tradition vornehmlich als staatliches Geschäft und die globale Ordnung in einem tradierten Sinne als westfälisches System souveräner Staaten begreift sowie dezidiert für ein solches Verständnis internationaler Politik wirbt.

Mouffes positive Attribuierung und Empfehlung einer multipolaren hegemonialen Konkurrenz verkennt, dass auf der internationalen Ebene konkurrierend-hegemoniale Ansprüche - aus strukturellen Gründen und im Unterschied zur agonal-einhegenden Situation innerhalb demokratischer Staaten und Gesellschaften - die Tendenz zur antagonistischen, potenziell immer auch gewaltförmigen und kriegerischen Realisierung vorweisen. Die Übertragung der Forderung nach agonaler Hegemonialkonkurrenz von der staatlichen auf die internationale Ebene steht insbesondere im Widerspruch zur hervorgehobenen Bedeutung, die Mouffe verbindlichen und durchsetzungsfähigen staatlichen Institutionen für die agonale Einhegung hegemonialer Konflikte zuspricht ${ }^{16}$ - und die in der internationalen Politik nicht in dieser Weise gegeben sind. Im Unterschied zu ihrer am Beispiel von liberaldemokratischen Gesellschaften erarbeiteten Hegemonietheorie sind außenpolitische und internationale Hegemonieansprüche in sehr viel stärkerem Maße an Ideen, Rechtfertigungen und

\footnotetext{
15 Vgl. Wojczewski (2018, S. 33), der im Anschluss an Laclau und Mouffe einen ,,hegemonic struggle over meanings and identities“ und einen ,shift in representational power in the field of IR" erkennt.

16 Vgl. erneut Mouffe (2007, S. 157, 2010, S. 37, 2014, S. 12, 14, 18, 23, 195); vgl. insgesamt Wallaschek (2017); Westphal (2019).
} 
Ziele einer globalen Mission ${ }^{17}$ sowie - nicht alleine, aber immer auch - an Potenziale der militärischen Durchsetzung zurückgebunden. So kann der von Mouffe (vgl. 2007, S. 149) im Anschluss an Gramsci geltend gemachte politische Stellungskrieg im internationalen, zwischenstaatlichen Raum kaum als agonal-befriedete Variante eines Antagonismus angesehen, sondern muss vielmehr als sein offener Ausbruch betrachtet werden. In diesem Sinne ist Mouffe gegenüber einzuwenden, dass die dem Bereich des Militärischen entlehnten Begriffe und Konzepte des Kampfes, der Strategie und des Stellungskrieges (vgl. 2007, S. 149), die ihr zufolge innerhalb grundsätzlich befriedeter Gesellschaften agonistisch verwirklicht werden können, durch ihre Rückübertragung ins Internationale gleichsam wieder ihre ursprüngliche, stärker materiale und militärische, gleichsam nichtmetaphorische Bedeutung annehmen.

In jüngster Zeit hat insbesondere Münkler die strukturelle Gefahr herausgestellt, die sich aus der Situation konkurrierender Hegemonialmächte ergibt. ${ }^{18}$ Wenngleich Münklers Ansatz sowohl in seinem realistisch grundierten Anspruch als auch in seiner Policy-Orientierung mit den Überlegungen Mouffes zur internationalen Politik korrespondiert, unterscheidet sich seine Beschreibung internationaler Hegemonialkonkurrenz aus strukturellen Gründen doch deutlich von Mouffes Modell einer multihegemonialen Ordnung. In seinen auch empirisch und historisch gesättigten Untersuchungen zum Verhältnis von Hegemonie und Imperialität hat Münkler auf der Grundlage einer Unterscheidung hegemonialer, imperialer und imperialistischer internationaler Strukturen und Politiken die destabilisierende und gewaltaffine Struktur hegemonialer Ansprüche in der internationalen Politik ausgewiesen. Im Rahmen systematischer Beobachtungen multipolarer hegemonialer Konkurrenzsituationen macht er hierfür insbesondere die Verbindung dreier Punkte geltend. So legt er dar, dass Hegemonialansprüche - im Unterschied zu unipolaren, nach Münkler imperialen Situationen - regemäßig im Plural vorhanden sind, dass sie unvermeidlich in sicherheitspolitische Rivalität zueinander geraten und dass sie schließlich mit dem Ziel der endgültigen Überwindung der Hegemonialkonkurrenz zu eigenen Gunsten einhergehen (vgl. 2006, S. 18, 67, 71-72, 75-77). Insbesondere Münklers Hinweis, dass internationale Hegemonieansprüche auf dem Selbstverständnis der eigenen Besonderheit und Einzigartigkeit fußen und das auch sicherheitspolitisch begründete Ziel der restlosen Beendigung der Konkurrenzsituation verfolgen, steht der von Mouffe geltend gemachten Vorstellung ,einer Vielzahl von Polen [...], die miteinander konkurrieren, ohne dass einer von ihnen den Anspruch erhebt, den anderen überlegen zu sein“ (2014, S. 73) diametral entgegen. Im Anschluss an Münkler, aber auch angesichts ihrer realistischen Sicht auf die internationale Politik, muss sich Mouffe die Frage stellen lassen, weshalb eine Hegemonialmacht den legitimen Bestand konkurrierender Hegemonialmächte anerkennen sollte - und weshalb sie, zuvörderst im Bereich der Sicherheitspolitik, Vertrauen darin setzen sollte, dass konkurrierende Hegemonialmächte ihr gegenüber diese Anerkennung erwidern. In Mouffes Forderung nach einer gewaltfreien, mithin wettbewerblich-agonalen Hege-

17 Vgl. hierzu Münkler (2006, S. 27, 132-134); vgl. auch Hausteiner und Huhnholz (2019, S. 12-13).

18 Eine Diskussion Mouffes in Bezug zu Hardt und Negri (vgl. v. a. 2001, S. 160-205) oder auch zu Deleuze und Guattari (vgl. v. a. 1973, III.7) wäre lohnend, kann hier aber nicht geleistet werden. 
monialkonkurrenz werden diese Punkte indes nicht thematisiert. Vielmehr blendet ihr hoffnungsvolles Modell wetteifernder Hegemonialmächte als ,freundschaftliche Feinde“ (2010, S. 30) bzw. - wie Mouffe dieses Konzept für die internationale Situation im Anschluss an Derrida benennt - als Beziehung der ,hostipitality“ (2014, S. 73; Hervorhebung dort) die Strukturen sowie die hieraus resultierenden Rationalitäten und Dynamiken multipolarer hegemonialer Konkurrenzsituationen und Rivalitäten aus. ${ }^{19}$

Die bisher ausgeführten Probleme einer Anwendung des agonistischen Politikmodells auf die internationale Politik resultieren insgesamt aus den unterschiedlichen Bedingungen, die in liberaldemokratischen Staaten einerseits und in der internationalen Politik andererseits gegeben sind. So ist zum einen festzustellen, dass wesentliche Voraussetzungen für die Entfaltung eines agonistischen Ansatzes, die in liberalen Demokratien vorliegen, auf der internationalen Ebene nicht bestehen. Zum anderen liegen in der internationalen Politik Strukturen, Dynamiken und auch Pfadabhängigkeiten vor, die auf der staatlichen Ebene nicht gegeben sind und daher berücksichtigt werden müssen. Gegen die Feststellung, dass die Schwierigkeiten der internationalen Adaption des agonistischen Modells aus unterschiedlichen Bedingungen auf der staatlichen und der internationalen Ebene resultieren, ließe sich freilich aus einer kosmopolitischen Perspektive einwenden, dass sie auf einer konzeptionell überwundenen Dichotomie von nationaler und internationaler Situation und Politik basiere. Nun aber macht Mouffe genau die Notwendigkeit dieser auch systematischen Unterscheidung dezidiert geltend. Es ist - paradoxerweise - also just das von Mouffe vertretene realistische Grundverständnis von internationaler Politik, das mit seiner Betonung der kategorialen und systematischen Unterschiedlichkeit zwischen nationaler und internationaler Situation einer Übertragung des agonistischen Modells von der Ebene des liberaldemokratischen Staates auf die internationale Sphäre entgegensteht.

Über die bisherige Diskussion hinausgehend, ist eine weitere, spezielle Implikation anzusprechen, die sich aus Mouffes Ausweis des demokratischen Paradoxes und aus ihrem Modell der agonistischen Demokratie für die Außenpolitik des liberaldemokratischen Staates und damit für sein Verhalten in der internationalen Politik ergibt. Diese Implikation, die man als inter-/nationales oder auch außen-/ innenpolitisches Paradox bezeichnen kann, besteht darin, dass das demokratische Paradox auf der Ebene der Außen- und zwischenstaatlichen Politik invers zur Ebene der innerstaatlichen Politik ausschlägt. Denn wenn Mouffe die Unüberwindbarkeit des demokratischen Paradoxes und des grundlegenden Dissenses zwischen dem liberalen und dem demokratischen Prinzip ausweist sowie angesichts der aktuellen Hegemonie des liberalen Prinzips die Forderung nach einem neuen egalitär-volksdemokratischen Projekt erhebt (vgl. 2007, S. 37-38, 71, 2010, S. 114-121, 124), stärkt dies in außenpolitischer und internationaler Hinsicht die andere, d. h. die liberale Seite des demokratischen Paradoxes. Durch das Plädoyer für die Seite der Gleichheit und die Stärkung der volksdemokratischen Tradition im Rahmen des demokratischen Paradoxes auf der staatlichen Ebene werden im außenpolitischen Verhalten des

19 Für eine auf die internationale Situation fokussierte Adaption der hostipitalité Derridas vgl. VaughanWilliams (2015, S. 144-147). 
Staates und im internationalen System eher die Aspekte der staatlichen Eigenständigkeit, der Unabhängigkeit, der Selbstbestimmung, der unbeschrankten staatlichen Souveränität, kurzum: die Seite der Freiheit des staatlichen Akteurs favorisiert. ${ }^{20}$ Der Emphase auf Gleichheit und Volksdemokratie auf der staatlichen Binnenebene entspricht in der Außen- und internationalen Politik strukturell die Betonung der souveränen Unabhängigkeit und Freiheit des Staates.

Zwar kann diese Umkehrung der Vorzeichen durch die internationale Wendung des demokratischen Paradoxes nicht als deterministischer Zusammenhang gelten. Gleichwohl geht dieser Zusammenhang über die Frage konkreter policies hinaus, sondern muss aus zweierlei Gründen als struktureller gelten. Erstens ist innerhalb des liberaldemokratischen Staates aufgrund des widerstreitenden Charakters von demokratischer Gleichheit und individueller Freiheit mit der Betonung und Hegemonialwerdung egalitär-demokratischer Prinzipen immer auch die Schwächung liberaler, universalistischer und kosmopolitischer Prinzipien verbunden - die im internationalen System allerdings eine wesentliche Grundlage für die Idee der völkerrechtlichen Gleichheit der Staaten bilden. Zweitens ist die Stärkung der egalitär-volksdemokratischen Seite innerhalb des demokratischen Paradoxes ja immer nur in Bezug auf den einzelstaatlichen Bereich und nur hinsichtlich des Staatsvolks des jeweiligen liberaldemokratischen Staates ein allgemeiner Anspruch, während er im internationalen Maßstab stets ein Partikularismus unter vielen ist. Was im Binnenverhältnis allgemein sein mag, bleibt im Außenverhältnis doch stets besonders. Auch eine hochgradig egalitäre und inklusive Definition des Volkes ist immer nur auf das jeweilige Volk bezogen (zu einem inklusiv-egalitären Populismus vgl. Mouffe 2018, Kap. 4). Diese beiden Punkte, die sich aus dem demokratischen Paradox ergeben, weisen darauf hin, dass das inverse Verhältnis zwischen egalitär-demokratischem und liberalem Prinzip auf der einzelstaatlichen und der zwischenstaatlichen Ebene nicht lediglich eine Frage konkreter policies, sondern strukturell angelegt ist. So folgt aus Mouffes Ausweis des demokratischen Paradoxes, dass der Betonung der volksdemokratischen Gleichheit auf der staatlichen Binnenebene in der Außen- und internationalen Politik strukturell die Betonung der souveränen Unabhängigkeit und Freiheit des Staates entspricht.

\section{Systemkonkurrenz und Pluralismus in Fragen der Demokratie und Menschenrechte}

Im Zentrum der Überlegungen Mouffes zur internationalen Politik steht der im vorstehenden Abschnitt diskutierte Ausweis einer agonalen, multipolaren Ordnung. Wie eingangs ausgewiesen, knüpft Mouffe dabei nicht an die vereinzelten Anschlüsse an ihr Denken in den IB oder an die Fruchtbarmachung ihres mit Laclau entwickelten Ansatzes in den $\mathrm{IB}^{21}$ an. Gleichwohl baut ihre Diskussion internationaler Hegemonie

\footnotetext{
${ }^{20}$ In einem strukturell analogen Sinne hinsichtlich der Unterscheidung zwischen innen- und außenpolitischem Bereich hat Niebuhr (1934, S. 91) festgestellt, dass ein ,paradox in patriotism“ darin bestehe, ,that patriotism transmutes individual unselfishness into national egoism“.

21 Siehe Fußnoten 5 und 6.
} 
doch implizit auf früheren Überlegungen zu hegemonialen Identitätskonstruktionen und Diskursen sowie zur Dekonstruktion universalistischer Ansprüche (vgl. hierzu Laclau und Mouffe 2001, S. 85-88, 95-96, 105-122; vgl. auch Laclau 1995; Howarth et al. 2000) auf und schließt diese gleichsam stillschweigend ein. Die fehlende explizite Thematisierung der Entstehung von kollektiven Identitäten und der Rolle von diskursiven Praktiken deutet nicht auf eine theoretische Wende hin, sondern spiegelt Mouffes Absicht wider, sich der Beschreibung und Kritik internationaler Politik nicht durch eine vornehmlich theoretisch-ontologische Analyse, sondern unmittelbar und empirisch sowie problem- und nicht zuletzt auch lösungsorientiert zuzuwenden.

In diesem praktischen Sinne nimmt Mouffe sich auch der Frage universalistischer Geltungsansprüche im internationalen System an, wobei die Kritik eines demokratischen und menschenrechtlichen Universalismus im Mittelpunkt ihrer Diskussion steht. Mit dem Ausweis und Vorschlag einer multipolaren Ordnung konkurrierender Hegemonien unmittelbar zusammenhängend, betont Mouffe die Notwendigkeit, die Gleichzeitigkeit und Inkommensurabilität unterschiedlicher politischer Systeme und Werteordnungen in der internationalen Politik anzuerkennen (vgl. 2014, S. 57). So hebt sie ausdrücklich hervor, dass eine globale multipolare Ordnung nicht ausschließlich aus Demokratien bestehen werde. Doch sei mit dem Ziel der Verhinderung extremer Antagonismen ,eine multipolare Welt selbst dann einer unipolaren vorzuziehen, wenn die regionalen Pole nicht demokratisch verfasst sind" (Mouffe 2014, S. 57). In der Forderung nach einer weltweiten Demokratisierung erkennt sie den letztlich kulturimperialistischen und illegitimen Versuch einer Universalisierung des westlichen Gesellschaftsmodells - der aus ihrer Sicht sowohl zum Scheitern verurteilt als auch gefährlich ist. Dabei sieht Mouffe nicht nur das liberaldemokratische Modell als "Ausdruck eines bestimmten kulturellen und historischen Kontextes“ (2014, S. 58) an, sondern gleichfalls die ,ihm eigen[e] Idee der Menschenrechte“ (2014, S. 58).

Die Anerkennung einer - wie man es in die Terminologie der IB übersetzen könnte - globalen Systemkonkurrenz sowie unterschiedlicher, kulturrelativer Vorstellungen von Menschenrechten muss sich Mouffe zufolge allerdings nicht zwingend von dem - allerdings fernen, zeitlich unbestimmten - Ziel der globalen Durchsetzung der Demokratie oder der weltweiten Wahrung von Menschenrechten verabschieden (vgl. 2014, S. 57-58, 62). Es gelte allerdings, die i.w.S. kulturelle und historische Pluralität von Vorstellungen und Formen der Demokratie und auch der Menschenrechte anzuerkennen, die auch stärker nichtsäkulare, gemeinschaftsorientierte Aspekte betonen oder auch die „Kompatibilität von Scharia und Demokratie“ (Mouffe 2014, S. 67, vgl. S. 61-70) einschließen würden. Einer hegemonialen Universalisierung westlicher Vorstellungen stellt Mouffe damit die Notwendigkeit der Anerkenntnis gegenüber, dass in anderen kulturellen Kontexten funktionale Äquivalente zu den westlich-kulturspezifischen Konstrukten der liberalen Demokratie und der Menschenrechte existieren und dass sich diese vom westlichen Modell auch erheblich unterscheiden können (vgl. 2009, S. 557-560, 2014, S. 59-62).

Mit Blick auf die Menschenrechtsthematik konkretisiert Mouffe dieses Argument, indem sie den Menschenrechten das Prinzip der Menschenwürde gegenüberstellt und betont, dass das in Europa und den USA vorherrschende Menschenrechtsverständnis 
als spezifisch westlich-individualistische Antwort auf die kulturübergreifende Notwendigkeit der Wahrung der Menschenwürde gelten könne (vgl. 2014, S. 60). Da Mouffe dies allerdings - auch in epistemologischer Hinsicht - nicht weiter ausführt, wird mit dieser Ersetzung der Menschenrechte durch die Menschenwürde das von ihr erkannte Problem der Universalisierung aber keineswegs gelöst, sondern nur vom Begriff und Konzept der Menschenrechte auf den Begriff und das Konzept der Menschenwürde verlagert. So ist hier einzuwenden, dass die Zurückweisung der Universalität der Menschenrechte zugunsten eines anderen Konzepts, das dann ebenfalls als interkulturelle Universalie fungiert, freilich nicht als Zurückweisung universalistischer Konzepte insgesamt gelten kann, sondern lediglich als Austausch einer Universalie durch eine andere. ${ }^{22}$

Wenngleich also auf diesem Wege die von Mouffe intendierte theoretische Überwindung des menschenrechtlichen Universalitätsanspruchs insgesamt nicht gelingt, scheint Mouffes Vorschlag der Menschenwürde doch aus einem anderen Grund für eine breite, interkulturelle Adaption geeignet zu sein. Denn obgleich die begriffliche Ersetzung von Menschenrechten durch Menschenwürde keinen theoretischen Vorteil liefert, schließt dies in pragmatischer Hinsicht und mit Blick auf die empirische Situation in der inter- und transnationalen Politik gleichwohl nicht aus, dass manche Ausdrücke und Redeweisen für interkulturelle Diskurse i.d. T. besser geeignet sein könnten als andere. Wenn Mouffe nun offen lässt, weshalb sich ausgerechnet das auf den ersten Blick überaus voraussetzungsreiche, aus der abendländischen philosophischen und theologischen Tradition stammende Konzept der Würde bzw. Dignitas als interkulturelle Grundlage für funktionale Äquivalente der Menschenrechte eignen sollte, dann lässt sich dies - zugegebenermaßen gegen den Strich - auch als Vorteil lesen und produktiv wenden. Denn genau eine solche Offenlassung erlaubt es, die interkulturelle Anschlussfähigkeit vielleicht nicht des Begriffs oder des Konzepts, aber doch der Rede von der Menschenwürde pragmatisch zu rechtfertigen. Da Mouffe auf die Idee der Menschenwürde in sehr unspezifischer Allgemeinheit verweist, verwendet sie den Ausdruck der Menschenwürde letztlich als einen Sammel- und Containerbegriff, der vielfältige und unterschiedlichste kulturspezifische Ausformulierungen von - nicht zwingend juridisch zu verstehenden - Menschen- und/oder Gruppenrechten ermöglicht (vgl. auch 2009, S. 557-558). So ist der Ausdruck der Menschenwürde von starker Assoziativität bei zugleich hohem normativen Gewicht. Die Rede von der Menschenwürde funktioniert inhaltlich weitgehend unbestimmt, dabei aber stets als Imperativ, der immer schon die Frage nach ihrer Realisierung aufwirft, die einer Antwort harrt. In diesem Sinne kann die Menschenwürde letzt-

\footnotetext{
22 Mouffe bedient sich immer wieder einer universalistischen wie auch essenzialistischen Sprache und Begrifflichkeit. Wenn Mouffe vom „Wesen des ,Politischen““ (2007, S. 15), von der „Natur des ,Politischen““ (2010, S. 128), von der „pluralistische[n] Natur der Welt des Sozialen“ (2007, S. 17) oder vom Politischen als Teil ,unserer ontologischen Verfassung“ (2007, S. 25) spricht, steht dies in einem deutlichen Spannungsverhältnis zu ihrem ,postfundamentalistischen ontologischen Bezugsrahme[n]“ (2019, S. 154) und ihrer Zurückweisung universalistischer und essenzialistischer Vorstellungen. Vgl. hierzu Marchart (2013, Kap. 9, 2010, S. 15-16); Kapoor (2002, S. 474-476); Wenman (2009, S. 113-114); FlügelMartinsen (2017, S. 180-184, 243-245); Wallaschek (2017, S. 5); Vasilache (2019b, S. 502-503). In der Konflikthaftigkeit des Sozialen besteht die essenzialistische Setzung eines letzten Grundes, ohne den auch Mouffe nicht auskommt.
} 
lich mit Laclau als leerer Signifikant gelten - der aber nun gerade aufgrund seiner inhaltlichen Leere bei gleichzeitiger normativer Assoziativität, gleichsam als Essenzialismus ohne Essenz, sich tatsächlich für eine breite, wenngleich freilich nicht universelle, interkulturelle Anschlussfähigkeit eignen könnte. ${ }^{23}$

Welche jeweils kulturspezifischen Antworten auf die Frage der Menschenwürde als angemessene und hinreichende gelten können und welche nicht, kann aus diesem Modell heraus zwar nicht entschieden werden. Dies aber entspricht zum Einen Mouffes Zurückweisung universaler Bewertungsmaßstäbe in der internationalen Politik. Zum Zweiten korrespondiert ein solcher Pluralismus in Fragen der Menschenrechte mit Mouffes Hinweis, dass ,eine Koexistenz gegensätzlicher politischer Systeme unvermeidlich ist“" (2014, S. 57). Denn auch in ihren kurzen Ausführungen zur Möglichkeit kultursensibler Formen von Demokratie und Menschenrechten plädiert Mouffe doch vor allem für eine dezidiert realpolitische Perspektivierung der internationalen Politik. So stehen ihre kurzen Ausführungen zu kulturspezifischen Formen von Demokratie und Menschenrechten im Kontext der allgemeinen und vagen Feststellung, dass man die „Möglichkeit, dass eines Tages die ganze Welt demokratisch sein könnte, [...] nicht verwerfen“ (Mouffe 2014, S. 57-58) müsse. Im Unterschied zu dieser langfristigen, zeitlich undefinierten Möglichkeit einer pluralistischen Äquivalenz unterschiedlicher Modelle von Demokratie und Menschenrechten erkennt Mouffe die vordringliche Aufgabe allerdings in der Herstellung einer multipolaren Ordnung konkurrierender Hegemonialmächte, in der die Existenz nichtdemokratischer Systeme und in der Folge auch ungleiche menschenrechtliche Schutzstandards hingenommen werden müssen.

Hieraus ergibt sich zugleich, dass der äquivalenten Daseinsberechtigung unterschiedlicher politischer Systeme noch eine weitere Bedeutungsdimension von Äquivalenz innewohnt und in der von Mouffe anvisierten multipolaren Ordnung angelegt ist. Denn so können die wenigen Hinweise auf eine kulturspezifisch globale Durchsetzung der Demokratie in unbestimmter Zukunft nicht darüber hinwegtäuschen, dass die von Mouffe favorisierte multihegemoniale Weltordnung ohne ein gewisses $\mathrm{Maß}$ an Äquivalenz auch im wörtlichen Sinne von Gleichgültigkeit gegenüber der Lebenssituation von Menschen in ebenso undemokratischen wie illiberalen Staaten nicht wird auskommen können. Daher wird die Forderung der Anerkenntnis, dass ,,in anderen Kulturen [...] Entscheidungsprozesse weniger individualistisch und kooperativer verlaufen“ (Mouffe 2014, S. 61), nicht nur die äquivalente Geltung von, sondern in praxi bis zu einem gewissen Grad immer auch die Gleichgültigkeit gegenüber undemokratischen und repressiven Modellen mit einschließen müssen. Diese bei Mouffe nicht thematisierte, aber aus ihren Überlegungen sich ergebende Konsequenz kann dabei als der normative Preis verstanden werden, der zur Abwendung der aus ihrer Sicht normativ sehr viel gravierenderen Konsequenzen eines

\footnotetext{
${ }^{23}$ Diese zugleich mit wie gegen Mouffe vorgeschlagene Wendung bzw. Fortentwicklung ihrer wenig ausführlichen Überlegungen fußt zugegebenermaßen auf einer „Freedom of Unfaithful Interpretation“ (Prozorov 2016, S. 14, vgl. S. 14-18). Zugleich scheint mir eine Fruchtbarmachung ihrer Überlegungen zur Menschenwürde als Essenzialismus ohne Essenz dem bei Mouffe festzustellenden Spannungsverhältnis bzw. Paradox zwischen der Zurückweisung essenzialistischer letzter Gründe einerseits und der gesetzten ontologischen Grundannahme der Konflikthaftigkeit des Sozialen andererseits (vgl. Fußnote 22) recht gut zu entsprechen.
} 
immer nur als hegemoniale Dominanz und letztlich als Imperialismus denkbaren Universalismus zu zahlen ist.

\section{Moralisierung der internationalen Politik}

Mouffe weist bereits in ihren Überlegungen zum demokratischen Paradox sowie in ihrem Entwurf der agonistischen Demokratie die Unangemessenheit und Gefahr einer Verhandlung politischer Fragen in vornehmlich moralischen Begriffen aus (vgl. 2010, S. 129, 2007, S. 11-12, 98-100, 2014, S. 30, 38-42, 51, 208-209). Sie stellt eine Moralisierung der Politik fest, die aus einer Verkennung des grundsätzlich konfliktiven Charakters des Politischen folge und sich aus deliberativen, konsensualen und kosmopolitisch-liberalen Vorstellungen des Politischen ergebe bzw. ihnen entspreche (vgl. Mouffe 2007, S. 99, 2014, S. 51, 59, 208-209). Im Zusammenhang ihrer Kritik am liberalen Interventionismus und ihres Vorschlags einer agonistischmultipolaren Weltordnung inklusive einer auch kulturspezifischen Systemheterogenität hebt sie nun hervor, dass der Moralisierung politischer Fragen in der internationalen Politik ein besonderes Eskalations- und Gefahrenpotenzial innewohne. Die Be- und Verhandlung internationaler Konflikte in moralischen Begriffen berge die Gefahr sowohl der Vervielfachung als auch der totalen Eskalation von Antagonismen und stehe daher ihrer agonistischen Einhegung entgegen (vgl. Mouffe 2007, S. 98-108). Wenn Widerstand gegen die unipolare Hegemonie des westlichliberalen Modells vornehmlich moralisch disqualifiziert und delegitimiert werde, erhöhe sich die Wahrscheinlichkeit gewaltförmigen Widerstandes, da widerständigen Bewegungen keine als legitim angesehene Möglichkeit der Herausforderung des hegemonialen Projektes mehr bleibe (vgl. Mouffe 2007, S. 107-108). Zugleich kritisiert Mouffe, dass eine übermäßige Moralisierung in der internationalen Politik die Gefahr der Dämonisierung des Gegners mit sich bringe, mit dem keinerlei Verhandlung, Kompromiss oder Ausgleich gesucht werden, sondern der nur noch als zu vernichtender Feind wahrgenommen werden könne, der „,beseitigt werden“ (2007, S. 99, vgl. auch S. 11-12) müsse.

Diese Überlegungen korrespondieren mit der schon bei Kant im sechsten Präliminarartikel des Traktats Zum ewigen Frieden formulierten Forderung, von allen „ehrlose[n] Stratagemen“ (1796, S. 12) abzusehen, die für einen künftigen Frieden zwischen den Kriegsparteien eine Bürde darstellen. Bereits Kant (1796, S. 12) hatte die Notwendigkeit hervorgehoben, kriegerische Auseinandersetzungen nicht in Begriffen totaler und unüberwindlicher Feindschaft auszutragen und von einer moralischen Verteufelung des Feindes abzusehen:

Denn irgend ein Vertrauen auf die Denkungsart des Feindes muß mitten im Kriege noch übrig bleiben, weil sonst auch kein Friede abgeschlossen werden könnte, und die Feindseligkeit in einen Ausrottungskrieg (bellum internecinum) ausschlagen würde [...].

Das Phänomen der moralischen Dämonisierung des Gegners hat, im theoretischen Rückgriff auf Kant, in jüngerer Zeit auch in den IB verstärkte Aufmerksamkeit erfahren. So ist im Rahmen von Forschungen zum liberalen Interventionismus und 
zum demokratischen Krieg die Neigung westlicher Regierungen diagnostiziert und diskutiert worden, die jeweiligen Gegner moralisch wie ethisch zu disqualifizieren und als das schlechthin Böse zu brandmarken. ${ }^{24}$ Die Tendenz einer Moralisierung internationaler Gegnerschaft scheint in demokratischen politischen Systemen und Gesellschaften strukturell sogar besonders naheliegend zu sein. Hierzu trägt nicht zuletzt der Umstand bei, dass in Demokratien ,[j]ustifications for war habitually draw on normative arguments“ (Freedman 2005, S. 94). Dass Regierungen in Demokratien die Zustimmung ihrer Bevölkerungen zu Kriegsbeteiligungen meist auch mit der Betonung moralisch-normativer Einsatzgründe zu erreichen suchen, ist dabei sowohl in theoretischen Auseinandersetzungen (vgl. Freedman 2005; Desch 2008; Ish-Shalom 2008; Vasilache 2019c, S. 148-152) diskutiert als auch in (komparativen) empirischen Arbeiten (vgl. Geis et al. 2010; Fey 2013; Becker-Jakob 2013) ausgewiesen worden. Die ,inclination to make inflationary use of the evilization frame“ (Müller 2014, S. 488) mit dem Ziel sowohl der Begründung von Militäreinsätzen als auch der Erzeugung von Zustimmung hierfür kann also als Ausdruck der hervorgehobenen Bedeutung normativer Rechtfertigungsmuster im liberalen Interventionismus gelten. Die im Kontext des „Liberalism of Imposition“ (Sørensen 2006, S. 251) entfalteten Begründungen für Kriegsteilnahmen - die freilich nicht grundsätzlich nur taktisch oder in der Sache unzutreffend sein müssen - reichen von der Beendigung von (bürger-)kriegerischen Auseinandersetzungen, der langfristigen Friedenssicherung und der Abwendung systematischer Menschenrechtsverletzungen, über die Stabilisierung von failing oder failed states sowie die Etablierung nationaler Selbstbestimmung in Sezessionsgebieten bis zur Absicht des Demokratieexports und zur Verhinderung der Verbreitung von Massenvernichtungswaffen.

Nun birgt die im liberalen Interventionismus seit 1990 festzustellende Disqualifizierung gegnerischer Akteure als hostis humani generis in der Tat die von Mouffe hervorgehobene Gefahr einer gewaltförmigen Eskalation von Antagonismen und Konflikten. Schon die Bezeichnung des Feindes der Menschheit suggeriert, dass mit einem solchen Akteur keine Koexistenz möglich, sondern vielmehr seine Beseitigung notwendig ist. Dabei kann eine Auseinandersetzung mit einem solchen Gegner sogar als Gefahrenabwehr und das eigene Kriegsengagement als moralische Pflicht verstanden und repräsentiert werden. Angesichts dessen kann schließlich auch geltend gemacht werden, dass völkerrechtliche Einschränkungen gegenüber einer solchen Pflicht zurückstehen müssen. In diesem Sinne sind im Rahmen des liberalen Interventionismus immer wieder rechtspolitische Bemühungen beteiligter Regierungen zu beobachten, die jeweiligen Gegner außerhalb des Kriegsvölkerrechts $\mathrm{zu}$ positionieren.

Mouffes Warnung vor einer Moralisierung der Politik steht nun aber ihr Plädoyer für eine affektive Aufladung der Auseinandersetzung zwischen kollektiven Identitäten gegenüber. Denn so sieht Mouffe die Existenz von „Leidenschaften“ (2019, S. 148) mit ihrer „Konnotatio[n] des Ungestümen“ (2019, S. 148), die es ,erlaubt, die Dimension des Konflikts hervorzuheben und von einer Konfrontation zwischen kollektiven politischen Identitäten zu sprechen“ (2019, S. 148), als wesentlich für

24 Vgl. Chandler (2014, S. 442); Geis und Wunderlich (2014); Hayden (2014); Müller (2014) sowie breit Schillings (2017). 
ein Modell agonistischer Politik an (vgl. i.d. S. auch 2000, S. 10, 12, 16-17; Laclau und Mouffe 2001, Kap. 3). Hinsichtlich ihrer Kritik der Moralisierung der Politik stellt sich daher die Frage, wie eine leidenschaftliche, affektive agonale Beziehung ohne den Aspekt der Moralisierung auskommen sollte - bzw. wie und durch wen das Ausmaß der Moralisierung zu verhandeln, zu entscheiden, zu implementieren und zu kontrollieren wäre. ${ }^{25}$ Zugleich ist mit Blick auf die Tendenz und Gefahr der Moralisierung von Konflikten grundsätzlich zu fragen, ob in der internationalen Politik die affektive Aufladung von kollektiven Identitäten und Wir-Sie-Unterscheidungen nicht generell eher in eine konfliktverschärfende denn -einhegende Richtung weist. Jedenfalls steht die Forderung Mouffes nach Berücksichtigung und Anerkenntnis einer affektiven Wir-Sie-Unterscheidung in einem Spannungsverhältnis zu ihrer Forderung nach der Entmoralisierung von Antagonismen bzw. der Politik insgesamt. Es handelt sich dabei um ein theoretisch-konzeptionelles Spannungsverhältnis, das überdies operational unklar bleibt.

Neben dem Plädoyer für die affektive und leidenschaftliche Konstitution kollektiver Identitäten weist Mouffes Kritik der Moralisierung allerdings noch einen weiteren, folgenreichen Unterschied zur Kritik der moralischen Dämonisierung internationaler Gegnerschaft in den IB auf. Denn auch wenn Mouffes Moralisierungskritik mit der Diagnose einer Tendenz zur Dämonisierung von gegnerischen Akteuren in den IB korrespondiert, besteht doch kein Einvernehmen darüber, ob diese Tendenz tatsächlich aus einer genuinen Moralisierung der Politik resultiert. Vielmehr spricht die in Demokratien oft kaum anders als normativ zu erreichende Zustimmung für militärische Einsätze für die Annahme, dass Regierungen sich auch aus instrumentellen Gründen vornehmlich normativ-moralischer Argumente für einen geplanten Waffengang bedienen. Eine solche Verwendung und Instrumentalisierung normativwertebasierter Diskurse zur Erzeugung von Zustimmung zu militärischen Einsätzen hat sich eindrücklich z. B. im Vorfeld des Irakkrieges von 2003 gezeigt. Der wesentliche und gewichtige Unterschied zwischen Mouffes Kritik und den Diagnosen zum liberalen Interventionismus in den IB besteht darin, dass diese in der Moralisierung des politischen Diskurses nicht zuletzt ein taktisches Instrument zum Zwecke der Begründung militärischer Eskalationen erkennen (vgl. i.d.S. z. B. Freedman 2005, S. 94; Chandler 2006, S. 53-88; Müller 2014, S. 488), während Mouffe gleichsam umgekehrt - die Moralisierung der Politik als Konsequenz der fehlenden Anerkennung des konflikthaften Charakters des Politischen und somit als Folge unterdrückter, negierter Antagonismen ansieht - und daher schließlich auch einer Ächtung des Krieges in der internationalen Politik skeptisch gegenübersteht.

\section{6 (Keine) Ächtung des Krieges}

Zwar spricht sich Mouffe nicht expressis verbis gegen die Ächtung des Krieges als Mittel der internationalen Politik aus. Doch ihre ausgeprägten Bedenken gegen eine auch völkerrechtlich verbindliche Kriegsächtung werden überaus deutlich und da-

25 Vgl. zur Diskussion von Emotionen in den IB Wolf (2012); Koschut (2015); Hutchison und Bleiker (2017). 
durch offenbar, dass sie sich zum Einen affirmativ auf jene Überlegungen Schmitts bezieht, in denen er eine Ächtung oder auch nur internationale Verrechtlichung des Krieges als Gefahr für das internationale System ablehnt (vgl. Mouffe 2007, S. 102, 104). Zum Zweiten kritisiert Mouffe unterschiedliche zeitgenössische Positionen, die eine Überwindung oder Verrechtlichung militärischer Gewalt beinhalten, just für den Versuch der Kriegsächtung - und weist diese Ansätze schließlich allesamt zurück (vgl. 2007, S. 78-79, 119, 129-130, 2014, S. 51-54). Ihre Skepsis gegen eine Ächtung des Krieges korrespondiert dabei zum Dritten auch damit, dass Mouffe Macht, Herrschaft und Gewalt nicht systematisch voneinander unterscheidet. Vielmehr beschreibt sie sie als verbundene, fließend ineinander übergehende Phänomene (vgl. Mouffe 2007, S. 119, 2010, S. 37, 125, 127-134), da ,die Natur des ,Politischen“" (Mouffe 2010, S. 128) und seine Machtfundierung zwingend auch in der „Anerkennung der Unauslöschbarkeit von Gewalt“ (Mouffe 2010, S. 128) bestehe. Die Gefahr der Ächtung des - man könnte sagen: regulären, interessengeleiteten Krieges besteht nach Mouffe nun darin, dass hierdurch Kriege nur noch als vorgeblich moralisch gerechte Kriege möglich seien. Diese aber tendierten dazu, letztlich schrankenlose Vernichtungskriege zu sein, da jede Gegnerschaft zur vermeintlich gerechten Sache als illegitim disqualifiziert werden könne. In der Folge könne, so stellt sie in Anlehnung an Schmitt (vgl. 2002, S. 37) fest, der Kriegsgegner als das absolut Böse und damit totaler Feind entmenschlicht und der bedingungslosen Auslöschung überantwortet werden (vgl. Mouffe 2007, S. 102-105).

Mit ihrer ausgeprägten Skepsis gegen eine völkerrechtliche Ächtung des Krieges handelt sich Mouffe nun allerdings ein empirisches und vor allem auch ein erhebliches theoretisches Problem für ihren Vorschlag eines internationalen agonistischen Modells ein. Zum Einen ist der empirische Einwand zu formulieren, dass die Vielzahl an grenzüberschreitenden gewaltförmigen Auseinandersetzungen seit 1990 gar keinen Anlass dafür gibt, eine effektive Ächtung des Krieges zu befürchten. Als Hindernis für die Entfaltung eines agonistischen Modells kann diese Sorge daher nicht gelten. Gewichtiger allerdings ist zum Zweiten eine begrifflich-definitorische Voraussetzung und theoretisch-konzeptionelle Implikation ihrer Argumentation, wonach die Ächtung des Krieges in der internationalen Politik die Möglichkeit agonaler Beziehungen untergrabe. Denn wenn Mouffe ausgerechnet in der Ächtung des Krieges ein Hindernis für die agonale Einhegung feindseliger Antagonismen erkennt, dann scheint sie selbst noch die militärische Auseinandersetzung dem Bereich der agonistischen Politik zuzurechnen. Dagegen aber stellt sich die Frage, ob die kriegerische Realisierung eines Konfliktes nicht vielmehr als seine antagonistische Eskalation gelten muss, um deren Verhinderung und Einhegung es Mouffe doch zu tun ist.

Bei Mouffe bleibt somit offen, gegen welche Form des internationalen Antagonismus und der Feindschaft agonistische Politik das Heilmittel sein soll, wenn selbst der Krieg noch zu ihrem Instrumentarium gehört, und welche Möglichkeiten einer weiteren, qualitativen Eskalation über den Krieg hinaus noch gegeben sein können und müssen, um von einem antagonistischen Konfliktmodus zu sprechen. Selbst wenn sich Kriege freilich in ihrer Länge und Intensität erheblich voneinander unterscheiden können, muss doch militärische Gewalt zwischen Staaten - nicht zuletzt unter Bedingungen hoch entwickelter Zerstörungs- und Tötungstechnologien - schon unter begrifflichen, konzeptionellen wie theoretischen Gesichtspunkten als letzte und 
schärfste Form der Austragung eines Konfliktes gelten. Und gerade unter Bezugnahme auf Mouffes Unterscheidung zwischen Antagonismus und Agonismus können im bewaffneten Kampf befindliche Parteien freilich weder als Mitbewerber oder Konkurrenten noch als agonistische Gegner angesehen, sondern letztlich nur in Begriffen antagonistischer, gleichsam echter Feindschaft verstanden werden. Während die Unterscheidung zwischen antagonistischer Konflikteskalation und ihrer agonistischen Einhegung den Kern ihres agonistischen Modells ausmacht, wird diese Unterscheidung just durch Mouffes Zurückweisung einer Ächtung des Krieges als reguläres Mittel der Politik verwischt. Sich gegen eine Ächtung des Krieges zu positionieren, untergräbt mit der Differenzierbarkeit zwischen antagonistischer Feindschaft und agonistischer Deeskalation den Vorschlag des agonistischen Modells selbst.

Nun scheinen mir allerdings die Bedenken Mouffes gegen eine völkerrechtliche Ächtung und Einhegung des staatlichen Rechts zum Kriege zwar mit ihrem Entwurf eines agonistischen Modells inkompatibel zu sein, aber aus ihrer Kritik der Moralisierung der internationalen Politik auch gar nicht zwingend zu folgen. Vielmehr resultiert Mouffes Skepsis gegen eine Beschränkung des Rechts zum Kriege aus der fehlenden Unterscheidung zwischen erstens der Legitimität des Krieges als Mittel der Politik (Ebene des ius ad bellum), zweitens seiner Durchführung bzw. Intensität (Ebene des ius in bello), drittens Phänomenen der Moralisierung von Konflikten und viertens Taktiken der Dämonisierung des Gegners. Vermeidet man hingegen die undifferenzierte Vermengung dieser - freilich miteinander verbundenen - Aspekte und Phänomene, dann ist nicht einzusehen, weshalb jede Form der auch moralischen Argumentation zu Politiken der Übermoralisierung und überdies zu Dämonisierungstaktiken führen sollte. Ebenso wenig lässt sich folgern, dass die Einschränkung des staatlichen ius ad bellum zu kriegerischer Enthemmung und zur Auflösung eines jeden ius in bello führen müsste. ${ }^{26}$ Und auch wenn Strategien und Taktiken der Dämonisierung des Gegners das Risiko entfesselter Kriegstechniken (d.h. einer Schwächung des ius in bello) bergen, kann doch der vermeintliche Gegenschluss, dass eine Normalisierung des Kriegs als Mittel der Politik (d.h. eine Schwächung des ius ad bellum) diese Gefahr reduziere, nicht überzeugen. In diesem Sinne zeigt sich, dass eine für das ius in bello tatsächlich tendenziell gefährliche Übermoralisierung von Konflikten keineswegs gegen eine Einschränkung des ius ad bellum, d.h. gegen eine fortschreitende Ächtung des Krieges als Mittel der Politik insgesamt, spricht.

Zugleich ist es möglich, das Problem einer übermäßigen Moralisierung in der internationalen Politik und die damit einhergehende Neigung zur Dämonisierung des Gegners zu adressieren, ohne einer Normalisierung des Krieges als Mittel der Politik das Wort zu reden. Hierfür ist eine weitere, doppelte Differenzierung geboten - die in Mouffes Überlegungen als Möglichkeit selbst schon angelegt ist. So ist zum

\footnotetext{
${ }^{26}$ Diese beiden Ebenen hängen zwar in vielfältiger Weise zusammen, sind aber zu unterscheiden. Dabei wird in der völkerrechtlichen Diskussion stärker die Unterscheidung und im moralphilosophisch orientierten Diskurs - und v. a. in der Diskussion zu Theorien des gerechten Krieges - stärker der Zusammenhang, gleichsam als „its two components“ (Murnion 2010, S. 23) betont. Vgl. mit Blick auf das Verhältnis zwischen ius ad bellum und ius in bello Guthrie und Quinlan (2007, S. 17-46). Vgl. lediglich überblicksweise zum Recht zum Kriege Damrosch und Scheffer (2018); O'Driscoll (2008) sowie zum Recht im Kriege Harvey et al. (2014). Vgl. auch noch immer Walzer (2015, S. 34-73).
} 
Einen die auf je konkrete, empirische Fälle zielende Zurückweisung der moralischen Dämonisierung des Gegners (und selbst des Feindes) von einer Ächtung des Krieges als einer allgemeinen Norm zu unterscheiden - wobei beides zugleich geltend gemacht werden kann. Dies würde nicht nur einer fragwürdigen Banalisierung des Krieges als eines gewöhnlichen und normativ unproblematischen politischen Instruments entgegenwirken, sondern ihn vielmehr als extremste Form antagonistischer Eskalation ausweisen - um deren Abwehr es Mouffe letztlich geht. Zum Zweiten ist die Frage der Moralisierung der internationalen Politik von der Frage ihrer Verrechtlichung zu unterscheiden. Denn wenngleich Mouffe diese beiden Dimensionen bzw. Phänomene in ihren Ausführungen zur internationalen Politik weitgehend gleichsetzt - wohl auch, weil sie ,die zunehmende Dominanz des Juridischen“ (2010, S. 113) als zur Moralisierung der Politik äquivalente Konsequenz der Verkennung des Politischen ansieht (vgl. 2010, S. 112-114) -, so folgen die von Mouffe ausgewiesenen Risiken der grenzenlosen Eskalation von Konflikten doch ausschließlich aus der Übermoralisierung der Politik, nicht allerdings aus ihrer Verrechtlichung. Diese Unterscheidung vorzunehmen, wäre innerhalb ihrer Argumentation möglich und dabei auch theoretisch konsequent. Denn in der weitgehenden Gleichsetzung der moralischen mit der rechtlichen Dimension besteht eine bemerkenswerte Übereinstimmung zwischen Mouffe und der von ihr als gefährlich zurückgewiesenen moralphilosophischen Vorstellung des gerechten Krieges. ${ }^{27}$ Berücksichtigt man indes die Unterscheidung zwischen moralischer und rechtlicher Dimension, steht die Kritik der enthemmenden und konfliktverschärfenden Wirkung einer „Politik im Register der Moral“ (Mouffe 2007, S. 95) sowie „der derzeitigen Verdrängung des Politischen durch das Ethische“ (Mouffe 2014, S. 39) einer völkerrechtlichen und dezidiert juridischen statt (alleine) moralischen Ächtung des Krieges keineswegs entgegen.

\section{Schlussbemerkung: Der normative Realismus Mouffes}

Der vorliegende Beitrag hatte eine Bestandsaufnahme und Diskussion des Nachdenkens Mouffes zur internationalen Politik zum Ziel. Dabei hat sich gezeigt, dass sich Mouffe auf globale Ordnungsfragen und damit letztlich auf die großen und mithin klassischen Themen der internationalen Politik und der IB konzentriert. Indem sie sich mit Uni- und Multipolarität, Hegemonialstreben, globaler Systemkonkurrenz, menschenrechtlichem Universalismus, Moral in der Politik und nicht zuletzt Konflikt und Krieg auseinandersetzt, werden grundsätzliche Herausforderungen und strukturelle Fragen der internationalen Politik adressiert. Während jeder dieser Themenund Fragekomplexe schon für sich genommen von besonderer Relevanz bzw. Einschlägigkeit ist, stehen diese Themen aber nicht nur einzeln oder nebeneinander im Zentrum ihrer Überlegungen. Mouffes spezieller Zugriff ergibt sich vielmehr aus der

\footnotetext{
27 Vgl. hierzu insgesamt Cohen (2018, insbes. auch S. 7-10) sowie Christopher (2004). Vgl. zu aktuellen Vorstellungen und Diskursen zum gerechten Krieg Evans (2005) sowie Bellamy (2006), zu aktuellen Herausforderungen O’Driscoll (2008), für eine postmoderne Perspektivierung Murnion (2010) und für eine Kritik des gerechten Krieges als Mythos Fiala (2008).
} 
Gesamtschau ihrer Ausführungen zur internationalen Politik. So zeichnet sich ihr Ansatz durch eine miteinander verflochtene Diskussion dieser Themenschwerpunkte im Lichte ihres agonistischen Politikmodells sowie einen konflikt- und machtanalytisch spezialisierten Blick aus. Mouffes Zugang zur internationalen Politik ist dadurch gekennzeichnet, dass er seinen Ausgang aus einem empirischen Interesse nimmt, welches zugleich nicht lediglich theoretisch beleuchtet, sondern theoretisch reflexiv konstituiert wird. In dieser Verschränkung von empirischem und theoretischem Zugriff drückt sich überdies ihr Anspruch aus, auch handlungsleitende Einsichten und konkrete Empfehlungen für die internationale Politik zu formulieren. Sie ist gleichermaßen an der Untersuchung internationaler Politik wie an der aus ihren Konzepten sich ergebenden Praxis interessiert. In ihren Überlegungen zur internationalen Politik sind die Ebenen der Analyse und der Anwendung unmittelbar aufeinander bezogen. Mit Blick auf diese auch in normativer Hinsicht folgenreiche Verschränkung von empirischer und theoretischer sowie analytischer und praktischer Perspektivierung sind nun abschließend drei allgemeinere Implikationen und Aspekte zu thematisieren, die sich aus Mouffes Sicht auf das Internationale ergeben. Diese betreffen erstens das Problem des methodologischen Nationalismus, zweitens die bemerkenswerte normative Wendung ihres realistischen Denkens sowie schließlich drittens die Frage nach einer internationalen politischen Theorie.

In der Diskussion der Überlegungen Mouffes zur internationalen Politik ist eine im weiteren Sinne, d.h. ohne Referenz auf bestimmte realistische Denkschulen, realistisch inspirierte Vorstellung des internationalen Systems und grenzüberschreitender Beziehungen offenkundig geworden. Eine auf den Grundbestand realistischen Denkens rekurrierende Konzeptualisierung internationaler Politik spiegelt sich dabei insbesondere auch in ihrem empirischen wie konzeptionellen Fokus auf den souveränen Staat als dem wesentlichen Akteur der internationalen Politik wider. ${ }^{28}$ Der methodologische Nationalismus Mouffes tritt zum Einen explizit darin hervor, dass sie Verträge - die gemeinhin zwischen staatlichen Regierungen geschlossen und idealtypisch anschließend von nationalen Parlamenten ratifiziert werden - als das einzige Instrument der internationalen Politik versteht, dem überhaupt ein gewisses Maß an Verlässlichkeit zukommt. Zum Zweiten ist ihre Kritik an globalistischen und kosmopolitischen Ansätzen nicht zuletzt auch eine Kritik an einer auf die (zivil-)gesellschaftliche, transnationale Ebene konzentrierten Perspektive grenzüberschreitender Beziehungen und Politik, der sie eine gefährliche Staats- und Souveränitätsvergessenheit vorwirft. Schließlich bewegt sich mit der Forderung eines multipolaren Gleichgewichts von Hegemonialmächten zum Dritten auch das Ergebnis ihrer Analyse innerhalb des systematischen und konzeptionellen Rahmens des methodologischen Nationalismus.

Einem methodologischen Nationalismus ist freilich entgegenzuhalten, dass sich grenzüberschreitende Beziehungen keineswegs alleine im Modell zwischenstaatlicher (Regierungs-)Beziehungen bewegen. Allerdings wäre es trotz ihrer realistischen, staatszentrierten Ausführungen voreilig, Mouffe eine Sicht zu attestieren, die nichtstaatlichen Akteuren jedwede Bedeutung abspräche. Eine solche Sicht wäre

28 Vgl. Niebuhr (1934); Carr (1964); Morgenthau (1993, S. 4-16); Waltz (2010, S. 95-97); Mearsheimer (2014, S. 17). 
auch kaum mit ihrer Diskurs- und Hegemonietheorie in Einklang zu bringen. Ihre auf zwischenstaatliche und intergouvernementale Beziehungen konzentrierte Optik kann vielmehr als thematische - und wohl auch theoriepolitische wie politischaktivistische - Schwerpunktsetzung gelten, die als Gegengewicht zu (zivil-)gesellschaftlichen und staatsvergessenen Ansätzen die fortgesetzte Bedeutung zwischenstaatlicher Beziehungsmodi betonen soll.

Wenn Mouffes Überzeugung, dass tradierte, im Wortsinne internationale Beziehungen weiterhin von hervorgehobener Bedeutung sind, einer realistischen Sicht auf das Internationale entspricht, gehen ihre Ausführungen in normativer Hinsicht allerdings über den Grundbestand des Realismus hinaus - und weisen sie dabei schließlich als normative Realistin aus. Mouffes Perspektive verdichtet sich zu einem normativen Realismus, in dem der normative und der realistische Blick und Anspruch nicht nur aufeinander bezogen sind, sondern sich letztlich reziprok bedingen. So versteht Mouffe die multipolare Konkurrenz hegemonialer Mächte nicht als Resultat der anarchischen Grundverfasstheit des internationalen Systems, sondern macht eine multipolare Hegemonialkonkurrenz vielmehr als intentional zu etablierendes Modell und als normatives Erfordernis geltend (vgl. explizit 2007, S. 151). Und selbst das Fehlen einer ,zentrale[n] Autorität“ (Mouffe 2014, S. 49) auf globaler Ebene wird bei ihr nicht nur als strukturelle Eigenschaft des internationalen Systems konstatiert, sondern dezidiert als politisches Ziel formuliert. Im Gegensatz zum für das realistische Denken charakteristischen (positivistischen) Anspruch einer Feststellung von Strukturmerkmalen des internationalen Systems besteht Mouffes Ansatz in einem normativen Plädoyer für ebendiese Strukturmerkmale. Was im Realismus Gegenstand der Beschreibung ist, findet sich in ihrem Ansatz als Forderung. Die konfliktive Grundstruktur multipolarer Hegemonialkonkurrenz ist bei Mouffe nicht vornehmlich ein realpolitisch zu handhabendes Ergebnis staatlichen Machtstrebens, sondern vielmehr ein normatives Ideal, das zum Zwecke der Einhegung antagonistischer Eskalationen angestrebt werden sollte. Damit entfalten Mouffes Überlegungen zur internationalen Politik gleichsam einen Realismus après la lettre, in dem nicht lediglich - wie oftmals gegen das realistische Denken eingewandt - das (vermeintlich) Faktische zum Normativen, d. h. aus einem Sein ein Sollen, wird, sondern das (aus ihrer Sicht noch nicht hinlänglich realisierte) Faktische als normatives Ziel geltend gemacht wird. Es lässt sich mithin in jenem Sinne von einem Realismus après la lettre sprechen, als dass sowohl auf realistische Logiken rekurriert wird als auch eine realistische Analyse und Politik - sowie der Aufbau korrespondierender Akteurs- und Machtstrukturen - normativ gefordert werden.

Es handelt sich hierbei freilich nicht um Normativität im Sinne einer ontologischen Fundierung in Letztbegründungen, die im Widerspruch stünde zum ,,postfundamentalistische[n] Denken des Politischen“ (Marchart 2013, S. 11) Mouffes, in dem ,[a]lle sozialen Verhältnisse [...] als kontingent erkannt“ (Marchart 2013, S. 11; Hervorhebung dort) worden sind. ${ }^{29}$ Es geht allerdings durchaus um Normativität im Sinne starker Handlungsanweisungen und -anleitungen sowie um Forderungen an die politische Praxis. Mouffes Ziel besteht in einem theoretisch wie politisch gerechtfertigten Sollen. Nicht zuletzt deshalb aber können Mouffes Überlegungen zur

\footnotetext{
29 Siehe allerdings erneut Fußnoten 22 und 23.
} 
internationalen Politik als ein Beitrag zur internationalen politischen Theorie gelten. Zwar geht es ihr im Unterschied zur vornehmlich im englischsprachigen Raum beheimateten Tradition der International Political Theory nicht um ethisch-normative Fragen oder gar um die moralphilosophische Grundlegung einer internationalen Ethik. Ganz im Gegenteil weist sie sowohl die Möglichkeit von Letztbegründungen als auch die Behandlung internationaler Fragen in Begriffen der Moral als gefährlich zurück. Zugleich aber bilden ihre Ausführungen weder lediglich einen Beitrag zur politischen Theorie noch alleine zur Theorie der IB. Vielmehr besteht ihr Ansatz in der Öffnung ihrer politischen Theorie und ihrer Theorie des Politischen für Fragen der internationalen Politik - und zwar in einer Öffnung, in der einerseits der empirische Fokus selbst schon theoretisch konstituiert wird und andererseits das theoretische Nachdenken durchgehend gegenständlich angeleitet ist. Dabei ist auch das Erkenntnisziel ein sowohl theoretisches wie empirisches. Ein solcher Ansatz verbindet die originären Zugriffe und Erkenntnisziele der politischen Theorie und der IB. Daher kann er im Sinne einer subdisziplinären Annäherung - wenngleich nicht Verschmelzung - der Horizonte von politischer Theorie und IB auch in systematischer Hinsicht als ein Beitrag zu einer genuinen internationalen politischen Theorie gelten.

Danksagung Für konstruktive Kritik und Hinweise möchte ich den anonymen Gutachter*innen sowie der PVS-Redaktion meinen Dank aussprechen. Für einen produktiven E-Mail-Austausch zu Mouffe zu Beginn des Arbeitsprozesses an diesem Artikel möchte ich Eva Herschinger danken. Oliver Flügel-Martinsen bin ich für produktive Gespräche und hilfreiche Kritik zu Dank verpflichtet.

Funding Open Access funding provided by Projekt DEAL.

Open Access Dieser Artikel wird unter der Creative Commons Namensnennung 4.0 International Lizenz veröffentlicht, welche die Nutzung, Vervielfältigung, Bearbeitung, Verbreitung und Wiedergabe in jeglichem Medium und Format erlaubt, sofern Sie den/die ursprünglichen Autor(en) und die Quelle ordnungsgemäß nennen, einen Link zur Creative Commons Lizenz beifügen und angeben, ob Änderungen vorgenommen wurden.

Die in diesem Artikel enthaltenen Bilder und sonstiges Drittmaterial unterliegen ebenfalls der genannten Creative Commons Lizenz, sofern sich aus der Abbildungslegende nichts anderes ergibt. Sofern das betreffende Material nicht unter der genannten Creative Commons Lizenz steht und die betreffende Handlung nicht nach gesetzlichen Vorschriften erlaubt ist, ist für die oben aufgeführten Weiterverwendungen des Materials die Einwilligung des jeweiligen Rechteinhabers einzuholen.

Weitere Details zur Lizenz entnehmen Sie bitte der Lizenzinformation auf http://creativecommons.org/ licenses/by/4.0/deed.de.

\section{Literatur}

Acharya, Amitav. 2014. The end of American world order. Cambridge: Polity Press.

Becker-Jakob, Una. 2013. Canada: standing on guard for international law and human security? In The militant face of democracy: liberal forces for good, Hrsg. Anna Geis, Harald Müller, und Niklas Schörnig, 160-195. Cambridge: Cambridge University Press.

Bellamy, Alexander J. 2006. Just wars: from Cicero to Iraq. Cambridge: Polity Press.

Çalkıvik, Asli. 2017. Poststructuralism and postmodernism in international relations. In Oxford Research Encyclopedia of International Studies. https://doi.org/10.1093/acrefore/9780190846626.013.102.

Campbell, David. 1998. Writing security: United States foreign policy and the politics of identity. Minneapolis: University of Minnesota Press.

Carr, Edward H. 1964. The twenty years' crisis, 1919-1939. New York: Harper. 
Chandler, David. 2006. From Kosovo to Kabul and beyond: human rights and international intervention. London: Pluto Press.

Chandler, David. 2014. Beyond good and evil: ethics in a world of complexity. International Politics 51(4):441-457.

Christopher, Paul. 2004. The ethics of war and peace: an introduction to legal and moral issues. London: Pearson.

Cohen, Sheldon M. 2018. Arms and judgement: law, morality, and the conduct of war in the twentieth century. Abindgon: Routledge.

Comtesse, Dagmar, Oliver Flügel-Martinsen, Franziska Martinsen, und Martin Nonhoff (Hrsg.). 2019. Radikale Demokratietheorie. Ein Handbuch. Berlin: Suhrkamp.

Conway, Janet, und Jakeet Singh. 2011. Radical democracy in global perspective: notes from the pluriverse. Third World Quarterly 32(4):689-706.

Czempiel, Ernst-Otto. 2004. Internationale Beziehungen: Begriff, Gegenstand und Forschungsabsicht. In Einführung in die internationale Politik, Hrsg. Manfred Knapp, Gert Krell, 2-28. München: Oldenbourg.

Damrosch, Lori F., und David J. Scheffer (Hrsg.). 2018. Law and force in the new international order. Abingdon: Routledge.

Deleuze, Gilles, und Félix Guattari. 1973. L'anti-œedipe. Paris: Minuit.

Derrida, Jacques. 1994a. Politiques de l'amitié. Paris: Galilée.

Derrida, Jacques. 1994b. Force de loi. Paris: Galilée.

Derrida, Jacques. 2003. Voyous. Deux essais sur la raison. Paris: Galilée.

Desch, Michael C. 2008. America's liberal illiberalism. International Security 32(3):7-43.

Diez, Thomas. 1999. Speaking 'Europe': the politics of integration discourse. Journal of European Public Policy 6(4):598-613.

Diez, Thomas. 2014. Bedeutungen und Grenzen: Anmerkungen zur Diskursforschung in den deutschsprachigen Internationalen Beziehungen. In Diskursforschung in den Internationalen Beziehungen, Hrsg. Eva Herschinger, Judith Renner, 381-398. Baden-Baden: Nomos.

Duncombe, Constance, und Tim Dunne. 2018. After liberal world order. International Affairs 94(1):25-42. Dunne, Tim, und Trine Flockhart (Hrsg.). 2013. Liberal world orders. Oxford: Oxford University Press.

Edkins, Jenny. 1999. Poststructuralism and international relations: bringing the political back in. Boulder: Lynne Rienner.

Edkins, Jenny, und Nick Vaughan-Williams (Hrsg.). 2009. Critical theorists and international relations. Abingdon: Routledge.

Evans, Mark (Hrsg.). 2005. Just war theory: a reappraisal. Edinburgh: Edinburgh University Press.

Fey, Marco. 2013. 'The right thing to do'? British interventionism after the cold war. In The militant face of democracy: liberal forces for good, Hrsg. Anna Geis, Harald Müller, und Niklas Schörnig, 89-123. Cambridge: Cambridge University Press.

Fiala, Andrew. 2008. The just war myth: the moral illusions of war. Lanham: Rowman \& Littlefield.

Flockhart, Trine. 2016. The coming multi-order word. Contemporary Security Policy 37(1):3-30.

Flügel-Martinsen, Oliver. 2017. Befragungen des Politischen. Subjektkonstitution - Gesellschaftsordnung - Radikale Demokratie. Wiesbaden: Springer VS.

Flügel-Martinsen, Oliver, und Oliver Marchart. 2014. Editorial der Gastherausgeber. Chantal Mouffe in der Diskussion. Zeitschrift für politische Theorie 5(2):197-202.

Freedman, Lawrence. 2005. The age of liberal wars. Review of International Studies 31(S1):93-107.

Geis, Anna, und Carmen Wunderlich. 2014. The good, the bad, and the ugly: comparing the notions of 'rogue' and 'evil' in international politics. International Politics 51(4):458-474.

Geis, Anna, Harald Müller, und Niklas Schörnig. 2010. Liberale Demokratien und Krieg: Warum manche kämpfen und andere nicht. Zeitschrift für Internationale Beziehungen 17(2):171-202.

Guthrie, Charles, und Michael Quinlan. 2007. Just war. The just war tradition: ethics in modern warfare. London: Bloomsbury.

Hardt, Michael, und Antonio Negri. 2001. Empire. Cambridge: Harvard University Press.

Harvey, Caroline, James Summers, und Nigel D. White (Hrsg.). 2014. Contemporary challenges to the laws of war. Cambridge: Cambridge University Press.

Hausteiner, Eva Marlene, und Sebastian Huhnholz. 2019. Ordnungen imperialer Macht. Eine Bestandsaufnahme. In Imperien verstehen, Hrsg. Eva Marlene Hausteiner, Sebastian Huhnholz, 9-38. BadenBaden: Nomos.

Hayden, Patrick. 2014. Systemic evil and the international political imagination. International Politics 51(4):424-440. 
Heil, Reinhard, Andreas Hetzel, und Dirk Hommrich (Hrsg.). 2011. Unbedingte Demokratie. Fragen an die Klassiker neuzeitlichen politischen Denkens. Baden-Baden: Nomos.

Herschinger, Eva. 2017. Hegemonie und Identität. Ernesto Laclaus Werk in den Internationalen Beziehungen. In Ordnungen des Politischen. Einsätze und Wirkungen der Hegemonietheorie Ernesto Laclaus, Hrsg. Oliver Marchart, 127-143. Wiesbaden: Springer VS.

Herschinger, Eva, und Judith Renner (Hrsg.). 2014. Diskursforschung in den Internationalen Beziehungen. Baden-Baden: Nomos.

Hetzel, Andreas (Hrsg.). 2017. Radikale Demokratie. Zum Staatsverständnis von Chantal Mouffe und Ernesto Laclau. Baden-Baden: Nomos.

Howarth, David, Aletta J. Norval, und Yannis Stavrakakis (Hrsg.). 2000. Discourse theory and political analysis: identities, hegemonies and social change. Manchester: Manchester University Press.

Hurrell, Andrew. 2009. On global order: power, values, and the constitution of international society. Oxford: Oxford University Press.

Hutchison, Emma, und Roland Bleiker. 2017. Emotions, discourse and power in world politics. International Studies Review 19(3):501-508.

Ikenberry, G. John. 2011. Liberal Leviathan: the origins, crisis, and transformation of the American world order. Princeton: Princeton University Press.

Ish-Shalom, Piki. 2008. Theorization, harm, and the democratic imperative: lessons from the politicization of the democratic-peace thesis. International Studies Review 10(4):680-692.

Jahn, Beate. 2018. Liberal internationalism: historical trajectory and current prospects. International Affairs 94(1):43-61.

Kant, Immanuel. 1796. Zum ewigen Frieden. Ein philosophischer Entwurf. Königsberg: Friedrich Nicolovius.

Kapoor, Ilan. 2002. Deliberative democracy or agonistic pluralism? The relevance of the Habermas-Mouffe debate for third world politics. Alternatives 27(4):459-487.

Koschut, Simon. 2015. Macht der Gefühle: Zur Bedeutung von Emotionen für die sozialkonstruktivistische Diskursforschung in den IB. Zeitschrift für Internationale Beziehungen 22(2):7-33.

Laclau, Ernesto. 1995. Subject of politics, politics of the subject. Differences 7(1):146-164.

Laclau, Ernesto, und Chantal Mouffe. 2001. Hegemony and socialist strategy: towards a radical democratic politics. London: Verso.

Little, Adrian, und Moya Lloyd (Hrsg.). 2009. The politics of radical democracy. Edinburgh: Edinburgh University Press.

Marchart, Oliver. 2010. Die politische Differenz. Zum Denken des Politischen bei Nancy, Lefort, Badiou, Laclau und Agamben. Berlin: Suhrkamp.

Marchart, Oliver. 2013. Das unmögliche Objekt. Eine postfundamentalistische Theorie der Gesellschaft. Frankfurt am Main: Suhrkamp.

Mearsheimer, John J. 2014. The tragedy of great power politics. New York: W.W. Norton.

Morgenthau, Hans J. 1993. Politics among nations. New York: McGraw-Hill.

Mouffe, Chantal. 2000. Deliberative democracy or agonistic pluralism. IHS Political Science Series, WP 72. Wien: IHS.

Mouffe, Chantal. 2007. Über das Politische. Wider die kosmopolitische Illusion. Frankfurt am Main: Suhrkamp.

Mouffe, Chantal. 2009. Democracy in a multipolar world. Millennium 37(3):549-561.

Mouffe, Chantal. 2010. Das demokratische Paradox. Wien: Turia+Kant.

Mouffe, Chantal. 2014. Agonistik. Die Welt politisch denken. Berlin: Suhrkamp.

Mouffe, Chantal. 2018. Für einen linken Populismus. Berlin: Suhrkamp.

Mouffe, Chantal. 2019. Die Affekte der Demokratie. In Wenn Demokratien demokratisch untergehen, Hrsg. Ludger Hagedorn, Katharina Hasewend, und Shalini Randeria, 147-162. Wien: Passagen.

Müller, Harald. 2014. Evilization in liberal discourse: from Kant's 'unjust enemy' to today's 'rogue state. International Politics 51(4):475-491.

Münkler, Herfried. 2006. Imperien. Die Logik der Weltherrschaft - vom Alten Rom bis zu den Vereinigten Staaten. Berlin: Rowohlt.

Münkler, Herfried. 2019. Imperiale Ordnung: Die Governance-Leistung von Imperien. In Imperien verstehen, Hrsg. Eva Marlene Hausteiner, Sebastian Huhnholz, 71-100. Baden-Baden: Nomos.

Murnion, William E. 2010. A postmodern view of just war. In Intervention, terrorism, and torture: contemporary challenges to just war theory, Hrsg. Steven P. Lee, 23-40. Dordrecht: Springer.

Nabers, Dirk. 2015. A poststructuralist discourse theory of global politics. Houndmills: Palgrave Macmillan.

Niebuhr, Reinhold. 1934. Moral man and immoral society. New York: Charles Scribner's Sons. 
Nonhoff, Martin. 2017. Antagonismus und Antagonismen - hegemonietheoretische Aufklärung. In Ordnungen des Politischen. Einsätze und Wirkungen der Hegemonietheorie Ernesto Laclaus, Hrsg. Oliver Marchart, 81-102. Wiesbaden: Springer VS.

O'Driscoll, Cian. 2008. The renegotiation of the just war tradition and the right to war in the twenty-first century. Houndmills: Palgrave Macmillan.

Plaxton, Michael. 2000. Deconstructing diplomacy: Derridean political theory and U.N. deal-making. Law and Critique 11(1):73-97.

Priester, Karin. 2014. Mystik und Politik. Ernesto Laclau, Chantal Mouffe und die radikale Demokratie. Würzburg: Königshausen \& Neumann.

Prozorov, Sergei. 2016. Foucault, freedom and sovereignty. Abingdon: Routledge.

Reus-Smit, Christian. 2005. Liberal hierarchy and the licence to use force. Review of International Studies 31(S1):71-92.

Rosenberg, Justin. 2016. International relations in the prison of political science. International Relations 30(2):127-153.

Rzepka, Vincent, und Grit Straßenberger. 2014. Für einen konfliktiven Liberalismus. Chantal Mouffes Verteidigung der liberalen Demokratie. Zeitschrift für politische Theorie 5(2):217-233.

Schillings, Sonja. 2017. Enemies of all humankind: fictions of legitimate violence. Hanover: Dartmouth College Press.

Schmitt, Carl. 2002. Der Begriff des Politischen. Berlin: Duncker \& Humblot.

Sørensen, Georg. 2006. Liberalism of restraint and liberalism of imposition: liberal values and world order in the new millennium. International Relations 20(3):251-272.

Stengel, Frank A., und Dirk Nabers. 2019. Symposium: the contribution of Laclau's discourse theory to international relations and international political economy. New Political Science 41(2):248-262.

Tambakaki, Paulina. 2009. Cosmopolitanism or agonism? Alternative visions of world order. Critical Review of International Social and Political Philosophy 12(1):101-116.

Tønder, Lars, und Lasse Thomassen (Hrsg.). 2014. Radical democracy: politics between abundance and lack. Manchester: Manchester University Press.

Trend, David (Hrsg.). 1996. Radical democracy: identity, citizenship, and the state. New York: Routledge.

Tsakatika, Myrto. 2007. Governance vs. politics: the European Union's constitutive 'democratic deficit. Journal of European Public Policy 14(6):867-885.

Vasilache, Andreas. 2019a. Security in the sovereignty-governmentality continuum. Cambridge Review of International Affairs 32(6):681-711.

Vasilache, Andreas. 2019b. Dissens/Konflikt/Kampf. In Radikale Demokratietheorie. Ein Handbuch, Hrsg. Dagmar Comtesse, Oliver Flügel-Martinsen, Franziska Martinsen, und Martin Nonhoff, 492-503. Berlin: Suhrkamp.

Vasilache, Andreas. 2019c. Fixing Missions: Überlegungen zu einem Typus des demokratischen Krieges zwischen liberalem Interventionismus und demokratischer Imperialität. In Imperien verstehen, Hrsg. Eva Marlene Hausteiner, Sebastian Huhnholz, 129-168. Baden-Baden: Nomos.

Vaughan-Williams, Nick. 2015. Europe's border crisis: biopolitical security and beyond. Oxford: Oxford University Press.

Wallaschek, Stefan. 2017. Chantal Mouffe und die Institutionenfrage. Zeitschrift für politische Theorie $8(1): 3-22$

Waltz, Kenneth N. 2010. Theory of international politics. Long Grove: Waveland Press.

Walzer, Michael. 2015. Just and unjust wars: a moral argument with historical illustrations. New York: Basic Books.

Wenman, Mark. 2009. Hegemony and globalist strategy. In The politics of radical democracy, Hrsg. Adrian Little, Moya Lloyd, 112-132. Edinburgh: Edinburgh University Press.

Westphal, Manon. 2019. Overcoming the institutional deficit of agonistic democracy. Res Publica 25(2):187-210.

Wojczewski, Thorsten. 2018. Global power shifts and world order: the contestation of 'western' discursive hegemony. Cambridge Review of International Affairs 31(1):33-52.

Wolf, Reinhard. 2012. Der ,emotional turn“ in den IB: Plädoyer für eine theoretische Überwindung methodischer Engführung. Zeitschrift für Außen- und Sicherheitspolitik 5(4):605-624.

Zehfuss, Maja. 2002. Constructivism in international relations: the politics of reality. Cambridge: Cambridge University Press.

Zürn, Michael. 2018. A theory of global governance: authority, legitimacy, and contestation. Oxford: Oxford University Press. 